\title{
Vacuum currents in braneworlds on AdS bulk with compact dimensions
}

\author{
S. Bellucci, ${ }^{a}$ A.A. Saharian ${ }^{b}$ and V. Vardanyan ${ }^{b}$ \\ ${ }^{a}$ INFN, Laboratori Nazionali di Frascati, \\ Via Enrico Fermi 40,00044 Frascati, Italy \\ ${ }^{b}$ Department of Physics, Yerevan State University, \\ 1 Alex Manoogian Street, 0025 Yerevan, Armenia \\ E-mail: bellucci@lnf.infn.it, saharian@ysu.am, vardanyanv@gmail.com
}

ABSTRACT: The two-point function and the vacuum expectation value (VEV) of the current density are investigated for a massive charged scalar field with arbitrary curvature coupling in the geometry of a brane on the background of AdS spacetime with partial toroidal compactification. The presence of a gauge field flux, enclosed by compact dimensions, is assumed. On the brane the field obeys Robin boundary condition and along compact dimensions periodicity conditions with general phases are imposed. There is a range in the space of the values for the coefficient in the boundary condition where the Poincare vacuum is unstable. This range depends on the location of the brane and is different for the regions between the brane and AdS boundary and between the brane and the horizon. In models with compact dimensions the stability condition is less restrictive than that for the AdS bulk with trivial topology. The vacuum charge density and the components of the current along non-compact dimensions vanish. The VEV of the current density along compact dimensions is a periodic function of the gauge field flux with the period equal to the flux quantum. It is decomposed into the boundary-free and brane-induced contributions. The asymptotic behavior of the latter is investigated near the brane, near the AdS boundary and near the horizon. It is shown that, in contrast to the VEVs of the field squared and energy-momentum tensor, the current density is finite on the brane and vanishes for the special case of Dirichlet boundary condition. Both the boundary-free and brane-induced contributions vanish on the AdS boundary. The brane-induced contribution vanishes on the horizon and for points near the horizon the current is dominated by the boundary-free part. In the near-horizon limit, the latter is connected to the corresponding quantity for a massless field in the Minkowski bulk by a simple conformal relation. Depending on the value of the Robin coefficient, the presence of the brane can either increase or decrease the 
vacuum currents. Applications are given for a higher-dimensional version of the RandallSundrum 1-brane model.

Keywords: Brane Dynamics in Gauge Theories, Field Theories in Higher Dimensions, AdS-CFT Correspondence

ARXIV EPRINT: 1508.07255 


\section{Contents}

1 Introduction $\quad 1$

2 Geometry of the problem and two-point functions 4

2.1 Set-up 4

2.2 Hadamard function in the R-region 6

$\begin{array}{ll}2.3 \text { Hadamard function in the L-region } & 11\end{array}$

3 Vacuum currents $\quad 14$

$\begin{array}{lll}3.1 & \text { R-region } & 15\end{array}$

3.2 L-region 23

$\begin{array}{lll}4 & \text { Conclusion } & 27\end{array}$

A Other representations of the two-point function 31

\section{Introduction}

Anti-de Sitter (AdS) spacetime is one of the simplest and most interesting spacetimes allowed by general relativity. It is the unique maximally symmetric solution of the vacuum Einstein equations with a negative cosmological constant (for geometrical properties of AdS space and its uses see, e.g., [1, 2]). Quantum field theory in AdS background has long been an active field of research for a variety of reasons. First of all, AdS spacetime has a high degree of symmetry and, because of this, numerous physical problems are exactly solvable in this geometry. The maximal symmetry of AdS simplifies many aspects of the study of quantum fields and the investigations of the corresponding field-theoretical effects may help to develop the research tools and insights to deal with more complicated geometries. Much of early interest to quantum field theory on AdS bulk was motivated by principal questions of the quantization of fields on curved backgrounds. The lack of global hyperbolicity and the presence of both regular and irregular modes give rise to a number of new features which have no analogues in quantum field theory on the Minkowski bulk. Being a constant negative curvature manifold, AdS space provides a convenient infrared regulator in interacting quantum field theories [3]. Its natural length scale can be used to regularize infrared divergences without reducing the symmetries. The importance of this theoretical research was increased by the natural appearance of AdS spacetime as a ground state in supergravity and Kaluza-Klein theories and also as the near horizon geometry of the extremal black holes and domain walls.

A further increase of interest is related to the crucial role of the AdS geometry in two exciting developments of the past decade such as the AdS/CFT correspondence and the 
braneworld scenario with large extra dimensions. The AdS/CFT correspondence [4-6] (see also [7]) represents a realization of the holographic principle and relates string theories or supergravity in the AdS bulk with a conformal field theory living on its boundary. It has many interesting consequences and provides a powerful tool for the investigation of gauge field theories. Among the recent developments of the AdS/CFT correspondence is the application to strong-coupling problems in condensed matter physics (familiar examples include holographic superconductors, quantum phase transitions and topological insulators). Moreover, the correspondence between the theories on AdS and Minkowski bulks may be used to derive new results in mathematical physics, in particular, in the theory of special functions (see, for instance, [8] and references therein). The braneworld scenario (for reviews see [9-12]) offers a new perspective on the hierarchy problem between the gravitational and electroweak mass scales. The main idea to resolve the large hierarchy is that the small coupling of four-dimensional gravity is generated by the large physical volume of extra dimensions. Braneworlds naturally appear in string/M-theory context and present intriguing possibilities to solve or to address from a different point of view various problems in particle physics and cosmology.

An inherent feature of all these models is that the boundary conditions on the fields should be specified in order to completely determine the dynamics. First of all the AdS spacetime is not globally hyperbolic and has a time-like future null infinity. As a consequence of this, the information may be lost to, or gained from, spatial infinity in finite coordinate time. In order to define a consistent quantum field theory, appropriate boundary conditions must be imposed [13-16]. The general class of allowed boundary conditions on the AdS boundary has been discussed in [17], based on the analysis of [18]. Different boundary conditions lead to different theories, in particular to different conformal field theory duals. In braneworld models on AdS bulk, the presence of branes gives rise to additional boundary conditions on the operator of a quantum field. These conditions depend on the specific geometry of the brane and have been discussed in [19-21] for models with $Z_{2}$ symmetry. Another type of boundary conditions is induced by the presence of compact spatial dimensions. The extra compact dimensions are an inherent feature of braneworld models arising from string and M-theories. In these models one needs also to specify the periodicity conditions along compact dimensions. Different conditions correspond to topologically inequivalent field configurations $[22,23]$. The nontrivial periodicity conditions lead to a number of interesting quantum field-theoretical effects, which include instabilities in interacting field theories, topological mass generation, and symmetry breaking.

All these types of boundary conditions, imposed on the field operator, modify the spectrum of the zero-point fluctuations of a quantum field. As a consequence, the vacuum expectation values (VEVs) of physical observables are shifted by an amount depending on the geometry of the boundary and on the type of the boundary condition. This general phenomena is known as the Casimir effect. It has important implications on all scales, from mesoscopic condensed matter physics to cosmology. Since the original research by Casimir [24], for the electromagnetic field in the geometry of two parallel conducting plates, many theoretical and experimental works have been done in this direction (for reviews see [25-30]). In braneworlds, the boundary conditions imposed on the bulk fields will give 
Casimir-type contributions to the vacuum energy and to the vacuum forces acting on the branes. The latter provide a natural mechanism for stabilizing the interbrane distance (radion) in Randall-Sundrum-type models. The Casimir energy gives a contribution to both the brane and the bulk cosmological constants and should be taken into account in the self-consistent formulation of the scenario. Motivated by these issues, the investigations of the Casimir energy and related forces on AdS bulk have attracted a great deal of attention (see, for instance, the references in [31]). The Casimir effect in higher-dimensional generalizations of the AdS spacetime with compact internal spaces has been discussed in [32-39].

An important physical characteristic of the vacuum state for charged fields is the expectation value of the current density. It carries information about the geometry and topology of the background space and is responsible for the backreaction of the quantum field, as a source in semiclassical Maxwell's equations. In the present paper we investigate the VEV of the current density for a charged scalar field in background of locally AdS spacetime with an arbitrary number of toroidally compactified spatial dimensions, in the presence of a brane parallel to the AdS boundary. The corresponding problem in the absence of the brane has been considered recently in [40] and here we shall be mainly concerned with the brane-induced effects. Both the zero and finite temperature expectation values of the current density for charged scalar and fermionic fields in background of flat spacetime with toral dimensions were investigated in [41-43]. Applications were given to the electronic subsystem of cylindrical and toroidal carbon nanotubes described in terms of a $(2+1)$-dimensional effective field theory. The vacuum current densities for charged scalar and Dirac spinor fields in de Sitter spacetime with toroidally compact spatial dimensions are considered in [44]. The influence of boundaries on the vacuum currents in topologically nontrivial spaces are studied in $[45,46]$ for scalar and fermionic fields. The effects of nontrivial topology induced by the compactification of a cosmic string along its axis have been discussed in [47-49].

The organization of the paper is as follows. In the next section we specify the bulk and boundary geometries under consideration and evaluate the Hadamard function for a charged massive scalar field in both regions on the right and on the left to the brane (referred to as R- and L-regions, respectively). The brane-induced contributions are manifestly extracted and they are presented in the form well suited for the investigation of the VEVs of local physical observables bilinear in the field. As such an observable, in section 3, we consider the current density. The corresponding VEVs are decomposed into boundaryfree and brane-induced contributions for both R- and L-regions. The asymptotic behavior of the brane-induced contributions is considered near the brane, near the AdS boundary and near the horizon. Limiting expressions are derived for small and large proper lengths of compact dimensions. The main results are summarized in section 4. In appendix we provide alternative representations for the Hadamard functions in the R- and L-regions. The expressions for the current densities obtained from these representations are used for the investigation of the near brane asymptotic. 


\section{Geometry of the problem and two-point functions}

\section{$2.1 \quad$ Set-up}

We consider a charged quantum scalar field $\varphi(x)$ with the mass $m$ and with the curvature coupling parameter $\xi$. In the presence of an external classical gauge field $A_{\mu}$, the corresponding field equation reads

$$
\left(g^{\mu \nu} D_{\mu} D_{\nu}+m^{2}+\xi R\right) \varphi(x)=0,
$$

where $D_{\mu}=\nabla_{\mu}+i e A_{\mu}$, $e$ is the charge of the field quanta, $\nabla_{\mu}$ is the operator of the covariant derivative associated with the metric tensor $g_{\mu \nu}$, and $R$ is the Ricci scalar for the background spacetime. The background geometry in the present paper is given by the interval

$$
d s^{2}=g_{\mu \nu} d x^{\mu} d x^{\nu}=e^{-2 y / a} \eta_{i k} d x^{i} d x^{k}-d y^{2},
$$

where $\eta_{i k}=\operatorname{diag}(1,-1, \ldots,-1)$ is the metric tensor for $D$-dimensional Minkowski spacetime, $i, k=0, \ldots, D-1$, and $\mu, \nu$ run from 0 to $D$. The local geometry described by $(2.2)$ coincides with that for $(D+1)$-dimensional AdS spacetime of the radius $a$, expressed in Poincaré coordinates. The corresponding metric tensor is a solution of the Einstein equations with a negative cosmological constant $\Lambda=-D(D-1) a^{-2} / 2$ and for the Ricci scalar one has $R=-D(D+1) / a^{2}$.

The spatial topology considered here will be different from that for AdS. Namely, we assume that the subspace with the coordinates $x^{l}, l=p+1, \ldots, D-1$, is compactified to a $q$-dimensional torus $T^{q}$ with $q=D-p-1$ (for a recent review of quantum filed-theoretical effects in toroidal topology see [50]). We shall denote by $L_{l}$ the length of the $l$ th compact dimension, $0 \leqslant x^{l} \leqslant L_{l}$. The ranges of the remaining coordinates are $-\infty<x^{l}<+\infty$, $l=1,2, \ldots, p$, and $-\infty<y<+\infty$. Consequently, for the subspace perpendicular to the $y$-axis we take the topology $R^{p} \times T^{q}$. Note that $L_{l}$ is the coordinate length of the compact dimension. For a fixed $y$, the proper length of the $l$ th compact dimension is given by $L_{(p) l}=e^{-y / a} L_{l}$ and it decreases with increasing $y$. The coordinate tarnsformation

$$
z=a e^{y / a}, 0 \leqslant z<\infty,
$$

brings the interval (2.2) into manifestly conformally flat form with the conformal factor $(a / z)^{2}$ :

$$
d s^{2}=(a / z)^{2}\left(\eta_{i k} d x^{i} d x^{k}-d z^{2}\right) .
$$

In terms of the new coordinate $z$, the AdS boundary and horizon are presented by the hypersurfaces $z=0$ and $z=\infty$, respectively. For the proper length, measured by an observer with a fixed coordinate $z$, one gets $L_{(p) l}=a L_{l} / z$.

We consider a field theory in a non-simply connected spacetime and for the complete formulation of the problem the periodicity conditions along compact dimensions should be specified. Here we impose the conditions

$$
\varphi\left(t, x^{1}, \ldots, x^{l}+L_{l}, \ldots, y\right)=e^{i \alpha_{l}} \varphi\left(t, x^{1}, \ldots, x^{l}, \ldots, y\right),
$$


for $l=p+1, \ldots, D-1$ with constant phases $\alpha_{l}$. In the literature, the most frequently considered special cases correspond to $\alpha_{l}=0$ (untwisted scalar) and $\alpha_{l}=\pi$ (twisted scalar). As we will see below, the nontrivial phases in the periodicity conditions give rise to the vacuum currents along compact dimensions. In the discussion below we shall assume that the gauge field $A_{\mu}$ is constant. Though the corresponding field strength vanishes, because of the nontrivial topology of the background spacetime, the VEVs of physical observables will be influenced by this sort of field configuration. This is an Aharonov-Bohm like effect of a constant gauge field. Let us consider two sets of the fields $\left(\varphi(x), A_{\mu}\right)$ and $\left(\varphi^{\prime}(x), A_{\mu}^{\prime}\right)$ connected by the gauge transformation $\varphi(x)=e^{-i e \chi(x)} \varphi^{\prime}(x), A_{\mu}=A_{\mu}^{\prime}+\partial_{\mu} \chi(x)$. For a constant gauge field, taking the function $\chi(x)=A_{\mu} x^{\mu}$, we see that in the new gauge the vector potential vanishes, $A_{\mu}^{\prime}=0$. But, after the gauge transformation the vector potential of the former gauge appears in the periodicity conditions for the new field operator:

$$
\varphi^{\prime}\left(t, x^{1}, \ldots, x^{l}+L_{l}, \ldots, y\right)=e^{i \tilde{\alpha}_{l}} \varphi^{\prime}\left(t, x^{1}, \ldots, x^{l}, \ldots, y\right),
$$

with $l=p+1, \ldots, D-1$, and with the new phases

$$
\tilde{\alpha}_{l}=\alpha_{l}+e A_{l} L_{l}
$$

In particular, nontrivial phases are generated for untwisted and twisted scalars. The phase shift in (2.6) is related to the magnetic flux $\Phi_{l}$ enclosed by the $l$ th compact dimension: $e A_{l} L_{l}=-2 \pi \Phi_{l} / \Phi_{0}$, where $\Phi_{0}=2 \pi / e$ is the flux quantum. Note that the gauge field fluxes play an important role in recent developments of string theory compactifications (for a review see [51]).

In the problem under consideration, the second type of boundary condition imposed on the field operator is induced by a brane parallel to the AdS boundary and located at $y=y_{0}$. The corresponding value for the conformal coordinate $z$ we shall denote by $z_{0}=a e^{y_{0} / a}$. On the brane we assume a gauge invariant boundary condition of the Robin type:

$$
\left(1+\beta n^{\mu} D_{\mu}\right) \varphi(x)=0, \quad y=y_{0},
$$

where $\beta$ is a constant and $n^{\mu}$ is the inward pointing normal to the brane. For the latter one has $n^{\mu}=\delta_{D}^{\mu}$ in the region $y>y_{0}$ and $n^{\mu}=-\delta_{D}^{\mu}$ in the region $y<y_{0}$. Note that, in general, the value of the coefficient $\beta$ for these regions could be different. The Robin boundary condition is a generalization of Dirichlet and Neumann conditions and naturally appears in a number of physical problems, including those in branworld scenario (see below). The spatial geometry under consideration for $D=2$, embedded into the 3dimensional Euclidean space, is displayed in figure 1. The compact dimension is presented by the circles and the thick circle corresponds to the location of the brane. We have also depicted the gauge field flux tube enclosed by the compact dimension. The proper length of the compact dimension decreases with increasing $y$.

The physical quantity we are interested in is the VEV of the current density, $\left\langle 0\left|j_{\mu}(x)\right| 0\right\rangle \equiv\left\langle j_{\mu}(x)\right\rangle$, for the field $\varphi(x)$, where $|0\rangle$ stands for the vacuum state. In quantum field theory on curved backgrounds the choice of the vacuum state is among the basic points. In what follows it will be assumed that the field is prepared in the Poincaré vacuum 


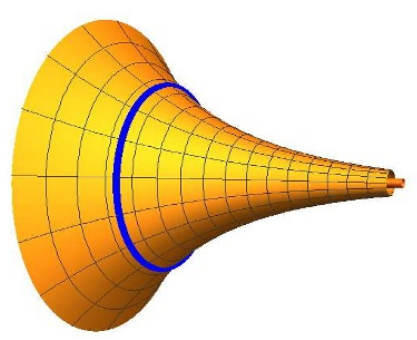

Figure 1. The spatial section of the geometry for $D=2$ embedded into a 3-dimensional Euclidean space. The thick circle corresponds to the brane.

state. The latter is relaized by the mode functions of the field which are obtained by solving the field equation in Poincaré coordinates (for the discussion of the relation between the Poincaré and global vacua see, for instance, [52]). The operator of the current density for a charged scalar field is defined by the expression

$$
j_{\mu}(x)=i e\left[\varphi^{+}(x) D_{\mu} \varphi(x)-\left(D_{\mu} \varphi^{+}(x)\right) \varphi(x)\right] .
$$

Its VEV is among the most important characteristics of the vacuum state. The procedure we shall use here for the evaluation of the expectation value is based on the formula

$$
\left\langle j_{\mu}(x)\right\rangle=\frac{i}{2} e \lim _{x^{\prime} \rightarrow x}\left(\partial_{\mu}-\partial_{\mu}^{\prime}+2 i e A_{\mu}\right) G\left(x, x^{\prime}\right),
$$

where

$$
\begin{aligned}
G\left(x, x^{\prime}\right) & =\left\langle 0\left|\varphi(x) \varphi^{+}\left(x^{\prime}\right)+\varphi^{+}\left(x^{\prime}\right) \varphi(x)\right| 0\right\rangle \\
& =\sum_{\sigma} \sum_{s= \pm} \varphi_{\sigma}^{(s)}(x) \varphi_{\sigma}^{(s) *}\left(x^{\prime}\right) .
\end{aligned}
$$

is the Hadamard function for the vacuum state under consideration. In (2.11), the summation goes over a complete orthonormal set of positive- and negative-energy mode functions $\varphi_{\sigma}^{( \pm)}(x)$ specified by a collective index $\sigma$ involving the corresponding quantum numbers. The mode functions obey the quasiperiodicity conditions (2.5) and the condition (2.8) on the brane.

So, as the first step, we shall evaluate the Hadamard function. Though the background AdS spacetime is homogeneous, the brane at $y=y_{0}$ has nonzero extrinsic curvature tensor and its sides are not equivalent. In particular, the VEVs differ in the regions on the right and on the left of the brane. The consideration requires different procedures for these regions and we discuss them separately. In what follows the regions $y>y_{0}$ and $y<y_{0}$ will be referred to as R-region (right region) and L-region (left region), respectively.

\subsection{Hadamard function in the R-region}

We shall work in the gauge with the fields $\left(\varphi^{\prime}(x), A_{\mu}^{\prime}=0\right)$, omitting the prime. In accordance with the problem symmetry, the mode functions can be factorized as

$$
\varphi_{\sigma}^{( \pm)}(x)=z^{D / 2} Z_{\nu}(\lambda z) e^{i k_{r} x^{r} \mp i \omega t},
$$


where the summation over $r$ in the exponent goes for $r=1, \ldots, D-1, Z_{\nu}(x)$ is a cylinder function of the order

$$
\nu=\sqrt{D^{2} / 4-D(D+1) \xi+m^{2} a^{2}}
$$

and

$$
\omega=\sqrt{\lambda^{2}+k^{2}}, k^{2}=\sum_{l=1}^{D-1} k_{l}^{2} .
$$

For imaginary values of the order $\nu$ the vacuum state becomes unstable [14-16] and in what follows we shall assume the values of the parameters for which $\nu \geqslant 0$. In the cases of conformally and minimally coupled massless fields one has $\nu=1 / 2$ and $\nu=D / 2$, respectively. In the former case, we have the standard conformal relation with the modes in the problem on Minkowski bulk with toral dimensions. In (2.12), for the components of the momentum one has $-\infty<k_{l}<+\infty, l=1, \ldots, p$, and

$$
k_{l}=\left(2 \pi n_{l}+\tilde{\alpha}_{l}\right) / L_{l}, l=p+1, \ldots, D-1
$$

with $n_{l}=0, \pm 1, \pm 2, \ldots$. The eigenvalues (2.15) for the components along compact dimensions are directly obtained from the quasiperiodicity conditions (2.6).

In the R-region, the function $Z_{\nu}(\lambda z)$ is a linear combination of the Bessel and Neumann functions, $J_{\nu}(\lambda z)$ and $Y_{\nu}(\lambda z)$. First we consider the case when for all the modes $\lambda$ is real. The changes in the evaluation procedure in the case when the modes with purely imaginary values of $\lambda$ are allowed (bound states) will be discussed below. The relative coefficient in the combination of the Bessel and Neumann functions is determined from the boundary condition (2.8) on the brane (with $D_{\mu}=\partial_{\mu}$ ) and one gets

$$
Z_{\nu}(\lambda z)=C_{\sigma} g_{\nu}\left(\lambda z_{0}, \lambda z\right)
$$

where, for the further convenience, we have introduced the function

$$
g_{\nu}(u, v)=J_{\nu}(v) \bar{Y}_{\nu}(u)-\bar{J}_{\nu}(u) Y_{\nu}(v)
$$

Here, for a given function $F(x)$, we use the notation

$$
\bar{F}(x)=A_{0} F(x)+B_{0} x F^{\prime}(x),
$$

with the coefficients

$$
A_{0}=1+\frac{D}{2} \delta_{y} \beta / a, \quad B_{0}=\delta_{y} \beta / a
$$

In (2.19) and in what follows, $\delta_{y}=1$ in the R-region and $\delta_{y}=-1$ in the L-region.

Now, the set of quantum numbers $\sigma$ is specified by $\sigma=\left(\lambda, \mathbf{k}_{p}, \mathbf{n}_{q}\right)$, where $\mathbf{k}_{p}=$ $\left(k_{1}, \ldots, k_{p}\right)$ is the momentum in non-compact space and $\mathbf{n}_{q}=\left(n_{p+1}, \ldots, n_{D-1}\right)$. The coefficient $C_{\sigma}$ is determined from the normalization condition

$$
\int d^{D} x g^{00} \sqrt{|g|} \varphi_{\sigma}^{(s)}(x) \varphi_{\sigma^{\prime}}^{\left(s^{\prime}\right) *}(x)=\frac{\delta_{s s^{\prime}}}{2 \omega} \delta\left(\lambda-\lambda^{\prime}\right) \delta\left(\mathbf{k}_{p}-\mathbf{k}_{p}^{\prime}\right) \delta_{\mathbf{n}_{q}, \mathbf{n}_{q}^{\prime}}
$$


where the $y$-integration goes over $\left[y_{0}, \infty\right)$. By using the mode functions (2.12) with the radial function from $(2.16)$, we find

$$
\left|C_{\sigma}\right|^{2}=\frac{a^{1-D} \lambda}{2(2 \pi)^{p} \omega V_{q}}\left[\bar{J}_{\nu}^{2}\left(\lambda z_{0}\right)+\bar{Y}_{\nu}^{2}\left(\lambda z_{0}\right)\right]^{-1},
$$

with $V_{q}=L_{p+1} \cdots L_{D-1}$ being the volume of the compact subspace.

Having determined the complete set of normalized modes, from the mode-sum in (2.11), for the Hadamard function one obtains the representation

$$
\begin{aligned}
G\left(x, x^{\prime}\right)= & \frac{\left(z z^{\prime}\right)^{D / 2}}{(2 \pi)^{p} a^{D-1} V_{q}} \sum_{\mathbf{n}_{q}} \int d \mathbf{k}_{p} e^{i k_{r} \Delta x^{r}} \int_{0}^{\infty} d \lambda \lambda \\
& \times \frac{\cos \left(\Delta t \sqrt{\lambda^{2}+k^{2}}\right)}{\sqrt{\lambda^{2}+k^{2}}} \frac{g_{\nu}\left(\lambda z_{0}, \lambda z\right) g_{\nu}\left(\lambda z_{0}, \lambda z^{\prime}\right)}{\bar{J}_{\nu}^{2}\left(\lambda z_{0}\right)+\bar{Y}_{\nu}^{2}\left(\lambda z_{0}\right)} .
\end{aligned}
$$

In order to extract explicitly the brane-induced contribution, we subtract from (2.22) the corresponding Hadamard function in the boundary-free AdS background which will be denoted by $G_{0}\left(x, x^{\prime}\right)$. The latter is obtained from (2.22) replacing the last fraction in the right-hand side by the product $J_{\nu}(\lambda z) J_{\nu}\left(\lambda z^{\prime}\right)$. By using the identity

$$
\frac{g_{\nu}\left(\lambda z_{0}, \lambda z\right) g_{\nu}\left(\lambda z_{0}, \lambda z^{\prime}\right)}{\bar{J}_{\nu}^{2}\left(\lambda z_{0}\right)+\bar{Y}_{\nu}^{2}\left(\lambda z_{0}\right)}=J_{\nu}(\lambda z) J_{\nu}\left(\lambda z^{\prime}\right)-\frac{1}{2} \sum_{j=1,2} \frac{\bar{J}_{\nu}\left(\lambda z_{0}\right)}{\bar{H}_{\nu}^{(j)}\left(\lambda z_{0}\right)} H_{\nu}^{(j)}(\lambda z) H_{\nu}^{(j)}\left(\lambda z^{\prime}\right)
$$

with $H_{\nu}^{(1,2)}(x)$ being the Hankel functions, the following decomposition is obtained

$$
\begin{aligned}
G\left(x, x^{\prime}\right)= & G_{0}\left(x, x^{\prime}\right)-\frac{a^{1-D}\left(z z^{\prime}\right)^{D / 2}}{2(2 \pi)^{p} V_{q}} \sum_{\mathbf{n}_{q}} \int d \mathbf{k}_{p} e^{i k_{r} \Delta x^{r}} \int_{0}^{\infty} d \lambda \\
& \times \lambda \frac{\cos \left(\Delta t \sqrt{\lambda^{2}+k^{2}}\right)}{\sqrt{\lambda^{2}+k^{2}}} \sum_{j=1,2} \frac{\bar{J}_{\nu}\left(\lambda z_{0}\right)}{\bar{H}_{\nu}^{(j)}\left(\lambda z_{0}\right)} H_{\nu}^{(j)}(\lambda z) H_{\nu}^{(j)}\left(\lambda z^{\prime}\right) .
\end{aligned}
$$

Now, assuming that $z>z_{0}$, we rotate the integration contour of the last integral by the angle $\pi / 2$ for $j=1$ and by $-\pi / 2$ for $j=2$. Introducing the modified Bessel functions $I_{\nu}(x)$ and $K_{\nu}(x)$, the following final expression is obtained:

$$
\begin{aligned}
G\left(x, x^{\prime}\right)= & G_{0}\left(x, x^{\prime}\right)-\frac{4\left(z z^{\prime}\right)^{D / 2}}{(2 \pi)^{p+1} a^{D-1} V_{q}} \sum_{\mathbf{n}_{q}} \int d \mathbf{k}_{p} e^{i k_{r} \Delta x^{r}} \\
& \times \int_{k}^{\infty} d u u \frac{\cosh \left(\Delta t \sqrt{u^{2}-k^{2}}\right)}{\sqrt{u^{2}-k^{2}}} \frac{\bar{I}_{\nu}\left(u z_{0}\right)}{\bar{K}_{\nu}\left(u z_{0}\right)} K_{\nu}(u z) K_{\nu}\left(u z^{\prime}\right),
\end{aligned}
$$

where the notation with overbar is defined by (2.18), (2.19) with $\delta_{y}=1$.

In deriving (2.25) we have assumed that for all the modes of the field $\lambda$ is real. In addition to these modes, bound states can be present. For them $\lambda$ is purely imaginary, $\lambda=i \eta, \eta>0$, and the mode functions have the form

$$
\varphi_{\sigma}^{( \pm)}(x)=C_{\sigma}^{(\mathrm{b})} z^{D / 2} K_{\nu}(\eta z) e^{i k_{r} x^{r} \mp i \omega(\eta) t}
$$


where $\omega(\eta)=\sqrt{k^{2}-\eta^{2}}$ (note that, in order to have normalizeability, we impose the regularity condition at the AdS horizon that excludes the solution with the modified Bessel function $I_{\nu}(\eta z)$ in the radial part). Let us denote by $k_{(q)}^{(0)}$ the lowest value of the momentum in the compact subspace. Assuming that $\left|\tilde{\alpha}_{i}\right| \leqslant \pi$, one has

$$
k_{(q)}^{(0) 2}=\sum_{i=p+1}^{D-1} \tilde{\alpha}_{i}^{2} / L_{i}^{2} .
$$

If $\eta>k_{(q)}^{(0)}$, then there are modes for which the energy is purely imaginary and the vacuum state is unstable. In order to have a stable vacuum, in what follows we assume that $\eta<k_{(q)}^{(0)}$. From the boundary condition (2.8) it follows that for bound states the possible values of $\eta$ are roots of the equation

$$
\bar{K}_{\nu}\left(\eta z_{0}\right)=0 .
$$

By using the recurrence relation for the Macdonald function, this equation can be rewritten as

$$
(\nu-D / 2-a / \beta) K_{\nu}(u)+u K_{\nu-1}(u)=0,
$$

with $u=\eta z_{0}$. From here it follows that there are no bound states for $a / \beta<\nu-D / 2$ (for the special mode in the case $a / \beta=\nu-D / 2$ see below). For $a / \beta>\nu-D / 2$ a single bound state $\lambda=i \eta$ appears. The corresponding root $\eta$ increases with increasing $a / \beta$ and for some critical value $\beta=\beta_{\mathrm{R}}=\beta_{\mathrm{R}}\left(k_{(q)}^{(0)}\right)$ one gets $\eta=k_{(q)}^{(0)}$. Here, $\beta_{\mathrm{R}}$ is the value of the Robin coefficient for which the root of the equation is equal to $k_{(q)}^{(0)}$. The stability of the vacuum state requires the condition $1 / \beta<1 / \beta_{\mathrm{R}}$. The critical value for the Robin coefficient depends on the lengths of the compact dimensions, on the phases in periodicity conditions and on the mass of the field through the parameter $\nu$. Note that for a brane on AdS bulk with all dimensions being non-compact one has $k_{(q)}^{(0)}=0$ and all the modes with $\lambda=i \eta$, $\eta>0$, lead to the instability. Hence, in models with compact dimensions the stability condition, in general, is less restrictive. Assuming that $a / \beta>\nu-D / 2$, let us denote by $u=u_{\nu}^{(R)}(a / \beta)$ the root of the equation (2.29). This root increases with increasing $a / \beta$ and does not depend on the location of the brane. The stability condition for the vacuum state is written as $u_{\nu}^{(R)}(a / \beta)<k_{(q)}^{(0)} z_{0}$. From here it follows that, for fixed values of the other parameters, when the brane approaches the AdS boundary ( $z_{0}$ decreases), started from the critical value $z_{0}=u_{\nu}^{(R)}(a / \beta) / k_{(q)}^{(0)}$, the vacuum in the R-region becomes unstable.

The coefficient $C_{\sigma}^{(\mathrm{b})}$ in (2.26) is found from the normalization condition (2.20) making the replacement $\delta\left(\lambda-\lambda^{\prime}\right) \rightarrow \delta_{\eta \eta^{\prime}}$. By using the result for the integral involving the square of the Macdonald function [53], one gets

$$
\left|C_{\sigma}^{(\mathrm{b})}\right|^{2}=-\frac{(2 \pi)^{-p} a^{1-D} \eta \bar{I}_{\nu}\left(\eta z_{0}\right)}{V_{q} \omega(\eta) z_{0} \bar{K}_{\nu}^{\prime}\left(\eta z_{0}\right)} .
$$

In deriving this expression we have used the relations

$$
K_{\nu}(x)=\frac{B_{0}}{\bar{I}_{\nu}(x)}, B_{0}^{2}\left(x^{2}+\nu^{2}\right)-A_{0}^{2}=B_{0} x \frac{\bar{K}_{\nu}^{\prime}(x)}{K_{\nu}(x)},
$$


valid for $x=\eta z_{0}$, with $\eta z_{0}$ being the solution of (2.28). These relations are obtained by making use of (2.28) and the Wronskian relation for the modified Bessel functions.

Now, in the presence of the bound state, the mode sum for the Hadamard function has two contributions. The first one comes from the modes with real $\lambda$ and is given by the expression (2.22). The second contribution comes from the bound state. For the latter, by using the corresponding mode functions (2.26) and the normalization coefficient from (2.30), one finds

$$
\begin{aligned}
G^{(\mathrm{b})}\left(x, x^{\prime}\right)= & -\frac{2 \alpha^{1-D}\left(z z^{\prime}\right)^{\frac{D}{2}}}{(2 \pi)^{p} V_{q} z_{0}} \sum_{\mathbf{n}_{q}} \int d \mathbf{k}_{p} e^{i k_{r} \Delta x^{r}} \eta \\
& \times \frac{\bar{I}_{\nu}\left(\eta z_{0}\right)}{\bar{K}_{\nu}^{\prime}\left(\eta z_{0}\right)} \frac{\cos (\omega(\eta) \Delta t)}{\omega(\eta)} K_{\nu}(\eta z) K_{\nu}\left(\eta z^{\prime}\right) .
\end{aligned}
$$

The evaluation of the part coming from the modes with real $\lambda$ is similar to that we have described above. The difference arises in the step when we rotate the integration contour in (2.24). Now, the integrand in this expression has poles $\lambda= \pm i \eta$ on the imaginary axis, where $\eta$ is the root of (2.28). After the rotation, the integration contour has to pass round these poles on the right by small semicircles. The integrals over the semicircles around $\lambda=i \eta$ and $\lambda=-i \eta$ give the residue at $\lambda=i \eta$ multiplied by $2 \pi i$. It can be seen that this residue term exactly cancels the contribution of the bound state in (2.32). Hence, we conclude that the expression (2.25) for the Hadamard function is valid in the case of the presence of bound states as well.

In addition to the modes discussed above, a mode may be present for which $\lambda=0$ and $\omega=k$. For this mode the function $Z_{\nu}$ in (2.12) is a linear combination of $z^{\nu}$ and $z^{-\nu}$. The part with $z^{\nu}$ is excluded by the normalizability condition and the mode functions have the form

$$
\varphi_{(\mathrm{R}) \sigma}^{( \pm)}(x)=C_{(\mathrm{R})} z^{D / 2-\nu} e^{i k_{r} x^{r} \mp i k t} .
$$

These modes are normalizable under the condition $\nu>1$ and for the coefficient one finds

$$
C_{(\mathrm{R})}^{2}=\frac{(\nu-1) z_{0}^{2 \nu-2}}{(2 \pi)^{p} V_{q} a^{D-1} k} .
$$

From the boundary condition on the brane it follows that the mode is allowed for the special value of the Robin coefficient determined from

$$
\beta / a=1 /(\nu-D / 2)
$$

For $\nu=D / 2$ this value corresponds to Neumann boundary condition and in this case the mode function does not depend on the coordinate $z$. An example of this special case is realized by a minimally coupled massless scalar field. Note that this special mode for a scalar field is the analog of the zero mode of the graviton in Randall-Sundrum 1-brane model [54].

For $\nu>1$ and in the case of Robin boundary condition with (2.35), the contribution of the special mode (2.33) should be separately added to the Hadamard function in formulas (2.22) and (2.24) (but not to (2.25), see below). Note that the mode function (2.33) 
can be written as $\varphi_{(\mathrm{R}) \sigma}^{( \pm)}(x)=\Omega_{(\mathrm{R})}(z) \varphi_{(\mathrm{M}) \sigma}^{( \pm)}(x)$, where

$$
\Omega_{(\mathrm{R})}(z)=\sqrt{2(\nu-1)} z_{0}^{\nu-1} \frac{z^{D / 2-\nu}}{a^{(D-1) / 2}}
$$

and $\varphi_{(\mathrm{M}) \sigma}^{( \pm)}(x)$ are the mode functions for a massless scalar field in $D$-dimensional Minkowski spacetime with the spatial topology $R^{p} \times T^{q}$. From here it follows that the contribution of the mode (2.33) to the Hadamard function is expressed as $G_{(\mathrm{R})}\left(x, x^{\prime}\right)=$ $\Omega_{(\mathrm{R})}(z) \Omega_{(\mathrm{R})}\left(z^{\prime}\right) G_{R^{p} \times T^{q}}^{(\mathrm{M})}\left(x, x^{\prime}\right)$, where $G_{R^{p} \times T^{q}}^{(\mathrm{M})}\left(x, x^{\prime}\right)$ is the corresponding function for a massless scalar field in $D$-dimensional Minkowski spacetime with the spatial topology $R^{p} \times T^{q}$. By using the expression for the latter one gets

$$
G_{(\mathrm{R})}\left(x, x^{\prime}\right)=\frac{2 \Omega_{(\mathrm{R})}(z) \Omega_{(\mathrm{R})}\left(z^{\prime}\right)}{(2 \pi)^{p+1 / 2} V_{q}} \sum_{\mathbf{n}_{q}} e^{i k_{l} \Delta x^{l}} \frac{K_{(p-1) / 2}\left(k_{(q)} s_{p}\right)}{\left(s_{p} / k_{(q)}\right)^{(p-1) / 2}} .
$$

where the summation in the exponent goes over $l=p+1, \ldots, D-1$. In this expression we have defined $s_{p}=\sqrt{\left|\Delta \mathbf{x}_{p}\right|^{2}-(\Delta t)^{2}}$ and

$$
k_{(q)}^{2}=\sum_{l=p+1}^{D-1} k_{l}^{2}=\sum_{l=p+1}^{D-1}\left(2 \pi n_{l}+\tilde{\alpha}_{l}\right)^{2} / L_{l}^{2}
$$

is the squared momentum in the compact subspace.

It can be seen that the expression (2.25) for the Hadamard function is not changed by the presence of the mode (2.33). Indeed, under the condition (2.35), the contribution (2.37) of this mode is separately added to the right-hand sides of (2.22) and (2.24). Now we should take into account that the integrand in (2.24) with separate $j$ has a simple pole at $\lambda=0$ and in the rotation of the integration contour this pole should be avoided by arcs of a circle of small radius. The contributions of the integrals over these arcs exactly cancel the contribution (2.37) of the special mode and, as a consequence of this, the representation (2.25) is not changed.

\subsection{Hadamard function in the L-region}

In the region between the brane and AdS boundary, $y<y_{0}$, the mode functions still have the form (2.12). From the normalizability condition it follows that for $\nu \geqslant 1$ we should take

$$
Z_{\nu}(\lambda z)=C_{\sigma} J_{\nu}(\lambda z)
$$

For the part of the solution with the Neumann function, the normalization integral diverges on the AdS boundary $z=0$. In AdS/CFT correspondence normalizable and nonnormalizable modes are dual to states and sources, respectively. The key feature of AdS spacetime is the presence of a timelike boundary at infinity where appropriate boundary conditions should be imposed in order to have well-defined dynamics [13-16]. In the problem at hand, for the unique quantization procedure, in the range $0 \leqslant \nu<1$, we need to specify the boundary condition on the AdS boundary. For a scalar field, the Dirichlet 
and Neumann boundary conditions are the most frequently used ones. The general class of allowed boundary conditions of the Robin type, has been discussed in [17]. Boundary conditions, that include tangential derivatives of the field on the AdS boundary, have been investigated in $[55,56]$. In these papers the boundary condition is implemented by adding to the action for a scalar field a part located on the boundary (surface term) which contains a boundary kinetic term. The presence of the latter modifies the standard Klein-Gordon inner product by adding a boundary term. As a result of this, by an appropriate choice of the surface action, the modes with non-Dirichlet boundary conditions on the AdS boundary can be made normalizable in the region $\nu \geqslant 1$. However, the lack of manifestly positive inner product may give rise to ghosts in the bulk theory $[55,56]$. Here, we choose $(2.39)$ for all values of $\nu \geqslant 0$ that corresponds to Dirichlet condition on the AdS boundary in the case $0 \leqslant \nu<1$ (note that the analytic continuation to the Euclidean section automatically selects this boundary condition $[57,58])$.

From the boundary condition on the brane at $y=y_{0}$ it follows that the eigenvalues of $\lambda$ are roots of the equation

$$
\bar{J}_{\nu}\left(\lambda z_{0}\right)=0
$$

where the notation with overbar is defined in accordance with (2.18) where now in (2.19) $\delta_{y}=-1$. Hence, in the L-region the spectrum for $\lambda$ is discrete. First we consider the case when all the roots of (2.40) are real. Let us denote by $x=\gamma_{n}, n=1,2, \ldots$, the positive zeros of the functions $\bar{J}_{\nu}(x)$. Then, for the eigenvalues of $\lambda$ one has $\lambda=\gamma_{n} / z_{0}$ (for a mode with purely imaginary $\lambda$ see below). The normalization coefficient is determined from (2.20), with the replacement $\delta\left(\lambda-\lambda^{\prime}\right) \rightarrow \delta_{n n^{\prime}}$ and with the $y$-integration over $\left(-\infty, y_{0}\right]$. By using the standard result for the integral involving the square of the Bessel function [53], we find

$$
\left|C_{\sigma}\right|^{2}=\frac{\lambda z_{0} T_{\nu}\left(\lambda z_{0}\right)}{(2 \pi)^{p} a^{D-1} V_{q} z_{0}^{2} \omega}
$$

with the notation

$$
T_{\nu}(x)=x\left[x^{2} J_{\nu}^{\prime 2}(x)+\left(x^{2}-\nu^{2}\right) J_{\nu}^{2}(x)\right]^{-1} .
$$

Note that in the latter expression we could substitute $x J_{\nu}^{\prime}(x)=-A_{0} J_{\nu}(x) / B_{0}$.

Plugging the mode functions into the mode-sum (2.11), for the Hadamard function we get the expression

$$
\begin{aligned}
G\left(x, x^{\prime}\right)= & \frac{2 a^{1-D}\left(z z^{\prime}\right)^{D / 2}}{(2 \pi)^{p} V_{q} z_{0}^{2}} \sum_{\mathbf{n}_{q}} \int d \mathbf{k}_{p} e^{i k_{r} \Delta x^{r}} \sum_{n} \gamma_{n} \\
& \times \frac{\cos \left(\Delta t \sqrt{\gamma_{n}^{2} / z_{0}^{2}+k^{2}}\right)}{\sqrt{\gamma_{n}^{2} / z_{0}^{2}+k^{2}}} T_{\nu}\left(\gamma_{n}\right) J_{\nu}\left(\gamma_{n} z / z_{0}\right) J_{\nu}\left(\gamma_{n} z^{\prime} / z_{0}\right) .
\end{aligned}
$$

This expression involves the roots $\gamma_{n}$ which are given implicitly and is not convenient for the further evaluation of the current density. In order to obtain more workable expression we apply to the series over $n$ the summation formula [59-62]

$$
\begin{aligned}
\sum_{n=1}^{\infty} T_{\nu}\left(\gamma_{n}\right) f\left(\gamma_{n}\right)= & \frac{1}{2} \int_{0}^{\infty} d u f(u)-\frac{1}{2 \pi} \int_{0}^{\infty} d u \frac{\bar{K}_{\nu}(u)}{\bar{I}_{\nu}(u)} \\
& \times\left[e^{-\nu \pi i} f(i u)+e^{\nu \pi i} f(-i u)\right]
\end{aligned}
$$


The part in the Hadamard function coming from the first term in the right-hand side of (2.44) gives the corresponding function in the geometry without the brane. As a result, we get the following decomposed representation

$$
\begin{aligned}
G\left(x, x^{\prime}\right)= & G_{0}\left(x, x^{\prime}\right)-\frac{4 a^{1-D}\left(z z^{\prime}\right)^{D / 2}}{(2 \pi)^{p+1}} \sum_{q} \int d \mathbf{k}_{p} e^{i k_{r} \Delta x^{r}} \int_{k}^{\infty} d u \\
& \times \lambda \frac{\cosh \left(\Delta t \sqrt{u^{2}-k^{2}}\right)}{\sqrt{u^{2}-k^{2}}} \frac{\bar{K}_{\nu}\left(u z_{0}\right)}{\bar{I}_{\nu}\left(u z_{0}\right)} I_{\nu}(u z) I_{\nu}\left(u z^{\prime}\right) .
\end{aligned}
$$

Comparing with (2.25), we see that the expressions for the brane-induced parts in the Hadamard function in the R- and L-regions are obtained from each other by the replacements $I_{\nu}(x) \rightleftarrows K_{\nu}(x)$ and with the replacement $\beta \rightarrow-\beta$ in the notations with overbars. Comparing with (2.43), we see the important advantages of the representation (2.45): (i) the contribution of the brane is manifestly separated, (ii) the explicit knowledge of the zeros $\gamma_{n}$ is not required, (iii) the integrand in (2.45) is monotonic instead of the oscillatory behavior in (2.43) and (iv) the representation (2.45) holds in the presence of the bound state and of the special mode (see below) as well. In particular, the second and third points are important in the numerical evaluation of the vacuum currents.

Depending on the value of the Robin coefficient $\beta$, the equation (2.40) can have purely imaginary roots $\lambda=i \eta, \eta>0$. In this case, for the stability of the vacuum state we should assume that $\eta<k_{(q)}^{(0)}$. From the boundary condition on the brane it follows that the allowed values for $\eta$ are roots of the equation $\bar{I}_{\nu}\left(\eta z_{0}\right)=0$ which is written in the explicit form as

$$
(D / 2+\nu-a / \beta) I_{\nu}(u)+u I_{\nu+1}(u)=0
$$

where $u=\eta z_{0}$. From here we conclude that the modes under consideration are absent in the case $a / \beta<D / 2+\nu$ (the special mode for the case $a / \beta=D / 2+\nu$ is discussed below). For $a / \beta>D / 2+\nu$, the equation (2.46) has a single positive solution, $u=u_{\nu}^{(\mathrm{L})}(a / \beta)$, which increases with increasing $a / \beta$. Started from the critical value of $a / \beta$, denoted here by $a / \beta_{\mathrm{L}}$ and determined from the condition $u_{\nu}^{(L)}\left(a / \beta_{\mathrm{L}}\right)=k_{(q)}^{(0)} z_{0}$, the vacuum becomes unstable. Note that the critical values $\beta_{\mathrm{R}}$ and $\beta_{\mathrm{L}}$ of the coefficient in Robin boundary condition are different for the $\mathrm{R}$ - and L-region. As a result of this, there are values of $\beta$ for which the vacuum is stable in the one region and unstable in the other. We see that, under the condition $a / \beta>D / 2+\nu$, when the location of the brane approaches the AdS boundary, started from the critical value $z_{0}=u_{\nu}^{(\mathrm{L})}(a / \beta) / k_{(q)}^{(0)}$, the vacuum state becomes unstable. The summation formula (2.44) is valid also in the presence of purely imaginary roots if we add to the left-hand side the contribution from the corresponding modes. This contribution has the form (2.43) with the replacement $\gamma_{n} \rightarrow i \eta z_{0}$ and omitting the summation over $n$. As a result, the representation (2.45) is valid in the presence of purely imaginary roots as well.

Similar to the case of the R-region, under the condition

$$
\beta / a=1 /(D / 2+\nu)
$$


there is a $\lambda=0$ mode with the mode function

$$
\varphi_{(\mathrm{L}) \sigma}^{( \pm)}(x)=C_{(\mathrm{L})} z^{D / 2+\nu} e^{i k_{r} x^{r} \mp i k t},
$$

where

$$
C_{(\mathrm{L})}^{2}=\frac{(\nu+1) a^{1-D}}{(2 \pi)^{p} V_{q} z_{0}^{2 \nu+2} k} .
$$

The contribution of this mode to the Hadamard function is obtained by taking into account that $\varphi_{(\mathrm{L}) \sigma}^{( \pm)}(x)=\Omega_{(\mathrm{L})}(z) \varphi_{(\mathrm{M}) \sigma}^{( \pm)}(x)$, with

$$
\Omega_{(\mathrm{L})}(z)=\sqrt{2(\nu+1)} a^{(1-D) / 2} \frac{z^{D / 2+\nu}}{z_{0}^{\nu+1}},
$$

and is obtained from (2.37) by the replacement $\Omega_{(\mathrm{R})}(z) \rightarrow \Omega_{(\mathrm{L})}(z)$. This contribution should be added to the right-hand side of (2.43). The representation (2.45) is not changed.

As it has been discussed in [56], the presence of the brane with sufficiently large $z_{0}$ can serve as a mechanism to banish the ghosts in the region $z>z_{0}$ for the models with $\nu>1$ and with deformed surface term in the action of a scalar field. For fixed $z_{0}$ and $\nu$, the corresponding boundary condition contains two parameters that are coefficients in the kinetic and potential terms of the boundary action. The detailed analysis of the boundary condition, in the space of these parameters, for the presence of tachionic modes is given in [56]. These tachionic modes correspond to the modes with $\eta>k_{(q)}^{(0)}$ we have discussed above. Recall that, in the present paper, on the AdS boundary we have imposed Dirichlet boundary condition and the bulk theory contains no ghosts.

\section{Vacuum currents}

By using the expressions for the Hadamard function, from formula (2.10) we can see that the VEVs of the charge density and of the components of the current density along noncompact dimensions vanish:

$$
\left\langle j^{l}\right\rangle=0, l=0,1, \ldots, p .
$$

Of course, the latter property for the spatial components is a direct consequence of the problem symmetry under the reflections $x^{l} \rightarrow-x^{l}$. For the component along the $l$ th compact dimension one finds the decomposition

$$
\left\langle j^{l}\right\rangle=\left\langle j^{l}\right\rangle_{0}+\left\langle j^{l}\right\rangle_{b}, l=p+1, \ldots, D-1,
$$

where $\left\langle j^{l}\right\rangle_{0}$ is the corresponding VEV in the absence of the brane and the part $\left\langle j^{l}\right\rangle_{b}$ is induced by the brane.

The contribution $\left\langle j^{l}\right\rangle_{0}$ is investigated in [40] and is given by the expression

$$
\begin{aligned}
\left\langle j^{l}\right\rangle_{0}= & \frac{4 e a^{-1-D} L_{l}}{(2 \pi)^{(D+1) / 2}} \sum_{n_{l}=1}^{\infty} n_{l} \sin \left(\tilde{\alpha}_{l} n_{l}\right) \sum_{\mathbf{n}_{q-1}} \cos \left(\sum_{i \neq l} \tilde{\alpha}_{i} n_{i}\right) \\
& \times q_{\nu-1 / 2}^{(D+1) / 2}\left(1+\sum_{i} n_{i}^{2} L_{i}^{2} /\left(2 z^{2}\right)\right)
\end{aligned}
$$


where $\mathbf{n}_{q-1}=\left(n_{p+1}, \ldots, n_{l-1}, n_{l+1}, \ldots, n_{D-1}\right)$, and

$$
q_{\alpha}^{\mu}(x)=\frac{e^{-i \pi \mu} Q_{\alpha}^{\mu}(x)}{\left(x^{2}-1\right)^{\mu / 2}},
$$

with $Q_{\alpha}^{\mu}(x)$ being the the associated Legendre function of the second kind. Near the AdS boundary, $z \rightarrow 0$, the current density (3.3) behaves as $z^{D+2 \nu+2}$ and near the horizon the leading term in the asymptotic expansion is given by $\left\langle j^{l}\right\rangle_{0} \approx(z / a)^{D+1}\left\langle j^{l}\right\rangle_{R^{p+1} \times T^{q}}^{(\mathrm{M})}$. Here,

$$
\left\langle j^{l}\right\rangle_{R^{p+1} \times T^{q}}^{(\mathrm{M})}=2 e L_{l} \frac{\Gamma((D+1) / 2)}{\pi^{(D+1) / 2}} \sum_{n_{l}=1}^{\infty} n_{l} \sin \left(\tilde{\alpha}_{l} n_{l}\right) \sum_{\mathbf{n}_{q-1}} \frac{\cos \left(\sum_{i \neq l} \tilde{\alpha}_{i} n_{i}\right)}{\left(\sum_{i} n_{i}^{2} L_{i}^{2}\right)^{(D+1) / 2}},
$$

is the VEV of the current density for a massless scalar field in Minkowski spacetime with spatial topology $R^{p+1} \times T^{q}$.

Similar to the case of the Hadamard function, we shall consider the brane-induced contribution in the VEVs of the current density for the R- and L-regions separately.

\subsection{R-region}

In the R-region the brane-induced contribution in (3.2) is obtained from the corresponding part of the Hadamard function in (2.25). By using the relation

$$
\int_{0}^{\infty} d\left|\mathbf{k}_{p}\right|\left|\mathbf{k}_{p}\right|^{p-1} \int_{k}^{\infty} \frac{u f(u) d u}{\sqrt{u^{2}-k^{2}}}=\frac{\sqrt{\pi} \Gamma(p / 2)}{2 \Gamma((p+1) / 2)} \int_{0}^{\infty} d u u^{p} f\left(\sqrt{u^{2}+k_{(q)}^{2}}\right),
$$

we find the expression

$$
\left\langle j^{l}\right\rangle_{b}=-\frac{e C_{p} z^{D+2}}{2^{p-1} a^{D+1} V_{q}} \sum_{\mathbf{n}_{q}} k_{l} \int_{k_{(q)}}^{\infty} d x x\left(x^{2}-k_{(q)}^{2}\right)^{\frac{p-1}{2}} \frac{\bar{I}_{\nu}\left(z_{0} x\right)}{\bar{K}_{\nu}\left(z_{0} x\right)} K_{\nu}^{2}(z x),
$$

with the notation

$$
C_{p}=\frac{\pi^{-(p+1) / 2}}{\Gamma((p+1) / 2)} .
$$

From (3.7) we see that the brane-induced contribution to the current density along the $l$ th compact dimension is an odd periodic function of the phase $\tilde{\alpha}_{l}$ with the period $2 \pi$ and an even periodic function of the remaining phases $\tilde{\alpha}_{i}, i \neq l$, with the same period. By taking into account the relation (2.7), we conclude that the VEV of the current density is a periodic function of the magnetic flux with the period equal to the flux quantum.

The charge flux through the $(D-1)$-dimensional spatial hypersurface $x^{l}=$ const, having the normal $n_{l}=a / z$, is given by the quantity $n_{l}\left\langle j^{l}\right\rangle$. From (3.7) it follows that the corresponding brane-induced contribution, $n_{l}\left\langle j^{l}\right\rangle_{b}$, depends on the lengths of compact dimensions and on the coordinate $z$ in the form of the ratios $L_{i} / z_{0}$ and $z / z_{0}$. The latter is expressed in terms of the proper distance from the brane, $y-y_{0}$, as $z / z_{0}=e^{\left(y-y_{0}\right) / a}$.

Let us first consider the flat spacetime limit of (3.7), corresponding to the limiting transition $a \rightarrow \infty$ for fixed values of $y$ and $y_{0}$. In this limit, the order of the modified Bessel functions in (3.7) is large. Changing the integration variable to $x \rightarrow \nu x$, we use the corresponding uniform asymptotic expansions (see, for instance, [63]). By taking into 
account that in the limit under consideration $z \approx a+y$ and $z_{0} \approx a+y_{0}$, to the leading order we get

$$
\left\langle j^{l}\right\rangle_{b} \approx \frac{e C_{p}}{2^{p} V_{q}} \sum_{\mathbf{n}_{q}} k_{l} \int_{\sqrt{k_{(q)}^{2}+m^{2}}}^{\infty} d x\left(x^{2}-k_{(q)}^{2}-m^{2}\right)^{\frac{p-1}{2}} e^{-2 x\left(y-y_{0}\right)} \frac{\beta x+1}{\beta x-1} .
$$

The expression in the right-hand side coincides with the boundary-induced part of the current density for the geometry of a single Robin plate in $(D+1)$-dimensional Minkowski spacetime with spatial topology $R^{p+1} \times T^{q}$ (see [46]).

For a conformally coupled massless field one has $\nu=1 / 2$ and the modified Bessel functions in (3.7) are expressed in terms of the elementary functions. In this case the expression for the total current density takes the form

$$
\left\langle j^{l}\right\rangle=(z / a)^{D+1}\left[\left\langle j^{l}\right\rangle_{R^{p+1} \times T^{q}}^{(\mathrm{M})}+\frac{e C_{p}}{2^{p} V_{q}} \sum_{\mathbf{n}_{q}} k_{l} \int_{k_{(q)}}^{\infty} d x\left(x^{2}-k_{(q)}^{2}\right)^{\frac{p-1}{2}} e^{-2 x\left(z-z_{0}\right)} \frac{\beta_{\mathrm{M}}^{+} x+1}{\beta_{\mathrm{M}}^{+} x-1}\right],
$$

where (the notation with the lower sign is employed below)

$$
\beta_{\mathrm{M}}^{ \pm}=\frac{\beta z_{0} / a}{1 \pm(D-1) \beta /(2 a)}
$$

The right-hand side of (3.10), divided by the conformal factor $(z / a)^{D+1}$, coincides with the current density in the corresponding problem on Minkowski bulk with the plate at $z=z_{0}$ (compare with (3.9)) on which the field obeys the Robin boundary condition (2.8) with the replacement $\beta \rightarrow \beta_{\mathrm{M}}^{+}$. The difference of the Robin coefficients in the two conformally coupled problems is related to the fact that this coefficient is not conformally invariant.

At large distances from the brane compared with the AdS curvature radius, $y-y_{0} \gg a$, one has $z \gg z_{0}$. In addition, assuming that $z \gg L_{i}$, we can see that the dominant contribution to the integral in (3.7) comes from the region near the lower limit and the contribution of the mode with a given $\mathbf{n}_{q}$ is suppressed by the factor $e^{-2 z k_{(q)}}$. Under the condition $\left|\tilde{\alpha}_{i}\right|<\pi$, assuming that all the lengths $L_{i}$ are of the same order, the main contribution comes from the term with $n_{i}=0, i=p+1, \ldots, D-1$, and to the leading order we find

$$
\left\langle j^{l}\right\rangle_{b} \approx-\frac{e z^{D-(p-1) / 2} \tilde{\alpha}_{l} k_{(q)}^{(0)(p-1) / 2}}{2^{p+1} \pi^{(p-1) / 2} a^{D+1} V_{q} L_{l}} \frac{\bar{I}_{\nu}\left(z_{0} k_{(q)}^{(0)}\right)}{\bar{K}_{\nu}\left(z_{0} k_{(q)}^{(0)}\right)} e^{-2 z k_{(q)}^{(0)}} .
$$

This asymptotic corresponds to points near the AdS horizon. As we have already mentioned, in this limit, for the boundary-free part one has $\left\langle j^{l}\right\rangle_{0} \approx(z / a)^{D+1}\left\langle j^{l}\right\rangle_{R^{p+1} \times T^{q}}^{(\mathrm{M})}$, where $\left\langle j^{l}\right\rangle_{R^{p+1} \times T^{q}}^{(\mathrm{M})}$ is the current density for a massless scalar field in $(D+1)$-dimensional Minkowski spacetime with spatial topology $R^{p+1} \times T^{q}$ (see (3.5)) and with the lengths of the compact dimensions $L_{i}, i=p+1, \ldots, D-1$. From here we conclude that near the horizon the boundary-free part dominates in the total VEV. 
For fixed values of $z$ and $L_{i}$, when the location of the brane tends to the AdS boundary, $z_{0} \rightarrow 0$, to the leading order, from (3.7) one finds

$$
\begin{aligned}
\left\langle j^{l}\right\rangle_{b} \approx & -\frac{4 e C_{p} z^{D+2} z_{0}^{2 \nu}}{2^{2 \nu+p} \nu \Gamma^{2}(\nu) a^{D+1} V_{q}} \frac{A_{0}+\nu B_{0}}{A_{0}-\nu B_{0}} \sum_{\mathbf{n}_{q}} k_{l} k_{(q)}^{2 \nu+p+1} \\
& \times \int_{1}^{\infty} d x x^{2 \nu+1}\left(x^{2}-1\right)^{(p-1) / 2} K_{\nu}^{2}\left(z k_{(q)} x\right)
\end{aligned}
$$

and the VEV vanishes as $z_{0}^{2 \nu}$.

Now, let us consider the limit when the length of the $l$ th dimension is much smaller than the lengths of the other compact dimensions, $L_{l} \ll L_{i}$. In this case, in (3.7) the dominant contribution to the sum over $n_{i}, i=p+1, \ldots, D-1, i \neq l$, comes from large values of $\left|n_{i}\right|$ and we can replace the summation by the integration in accordance with

$$
\sum_{\mathbf{n}_{q-1}} f\left(k_{(q-1)}\right) \rightarrow \frac{2^{2-q} \pi^{-(q-1) / 2} V_{q}}{L_{l} \Gamma((q-1) / 2)} \int_{0}^{\infty} d u u^{q-2} f(u),
$$

where $k_{(q-1)}^{2}=k_{(q)}^{2}-k_{l}^{2}$. By making this replacement in (3.7), instead of $x$ we introduce a new integration variable $w$ according to $x=\sqrt{w^{2}+u^{2}+k_{l}^{2}}$. Then, introducing polar coordinates in the plane $(u, w)$, the integral over the angular variable is expressed in terms of the gamma function. As a result, to the leading order we get

$$
\left\langle j^{l}\right\rangle_{b} \approx-\frac{e C_{D-2} z^{D+2}}{2^{D-3} a^{D+1} L_{l}} \sum_{n_{l}=-\infty}^{+\infty} k_{l} \int_{\left|k_{l}\right|}^{\infty} d x x\left(x^{2}-k_{l}^{2}\right)^{\frac{D-3}{2}} \frac{\bar{I}_{\nu}\left(z_{0} x\right)}{\bar{K}_{\nu}\left(z_{0} x\right)} K_{\nu}^{2}(z x) .
$$

The expression in the right-hand side coincides with the brane-induced contribution in the model with a single compact dimension of the length $L_{l}(q=1, p=D-2)$.

If in addition to $L_{l} \ll L_{i}$ one has $L_{l} \ll z_{0}$, the arguments $z_{0} x$ of the modified Bessel functions in (3.15) are large. By using the corresponding asymptotic expressions [63], after the integration over $x$ we find

$$
\left\langle j^{l}\right\rangle_{b} \approx \frac{\left(1-2 \delta_{0 B_{0}}\right) e(z / a)^{D+1}}{2^{D-2} \pi^{D / 2} L_{l}\left(z-z_{0}\right)^{D / 2-1}} \sum_{n_{l}=-\infty}^{+\infty} k_{l}\left|k_{l}\right|^{D / 2-1} K_{D / 2-1}\left(2\left(z-z_{0}\right)\left|k_{l}\right|\right) .
$$

Here, for non-Dirichlet boundary conditions we have assumed that $|\beta| / a \gg L_{l} / z_{0}$. From (3.16) it follows that the brane-induced contribution is located near the brane within the region $z-z_{0} \lesssim L_{l}$ and has opposite signs for Dirichlet and non-Dirichlet boundary conditions. At distances $z-z_{0} \gg L_{l}$ it is suppressed by the factor $e^{-2\left(z-z_{0}\right) \tilde{\alpha}_{l} / L_{l}}$. Note that, in the limit $L_{l} \ll L_{i}, z$, for the boundary-free part one has the asymptotic

$$
\left\langle j^{l}\right\rangle_{0} \approx \frac{2 e \Gamma((D+1) / 2)}{\pi^{(D+1) / 2}(a / z)^{D+1} L_{l}^{D}} \sum_{n=1}^{\infty} \frac{\sin \left(\tilde{\alpha}_{l} n\right)}{n^{D}} .
$$

The expression (3.7) is not convenient for the investigation of the current density behavior near the brane. To this aim, a more convenient expression for the VEV of the 
current density is obtained by using the representation (A.3) for the Hadamard function. After the integrations over $w$ and $\mathbf{k}_{p}$, we get the following result

$$
\begin{aligned}
\left\langle j^{l}\right\rangle= & \frac{4 e a^{-D-1} z^{D+2}}{(2 \pi)^{p / 2+1} V_{q} L_{l}^{p}} \sum_{n=1}^{\infty} \frac{\sin \left(n \tilde{\alpha}_{l}\right)}{n^{p+1}} \sum_{\mathbf{n}_{q-1}} \int_{0}^{\infty} d \lambda \lambda \\
& \times \frac{g_{\nu}^{2}\left(\lambda z_{0}, \lambda z\right)}{\bar{J}_{\nu}^{2}\left(\lambda z_{0}\right)+\bar{Y}_{\nu}^{2}\left(\lambda z_{0}\right)} g_{p / 2+1}\left(n L_{l} \sqrt{\lambda^{2}+k_{(q-1)}^{2}}\right)
\end{aligned}
$$

where we have defined the function

$$
g_{\nu}(x)=x^{\nu} K_{\nu}(x)
$$

In the presence of a bound state, its contribution should be added to (3.18) separately. In the model with a single compact dimension $x^{l}$ of the length $L$ and with the phase $\tilde{\alpha}$, from (3.18) one finds

$$
\begin{aligned}
\left\langle j^{l}\right\rangle= & \frac{4 e a^{-D-1} z^{D+2}}{(2 \pi)^{D / 2} L^{D-1}} \sum_{n=1}^{\infty} \frac{\sin (n \tilde{\alpha})}{n^{D-1}} \int_{0}^{\infty} d \lambda \\
& \times \lambda \frac{g_{\nu}^{2}\left(\lambda z_{0}, \lambda z\right) g_{D / 2}(n L \lambda)}{\bar{J}_{\nu}^{2}\left(\lambda z_{0}\right)+\bar{Y}_{\nu}^{2}\left(\lambda z_{0}\right)}
\end{aligned}
$$

where we have substituted $p=D-2$.

An important result which follows from (3.18) is that the VEV of the current density is finite on the brane. The corresponding value is directly obtained from (3.18) putting $z=z_{0}$ and by taking into account that $g_{\nu}(u, u)=2 B_{0} / \pi$. For Dirichlet boundary condition both the current density and its normal derivative vanish on the brane. The finiteness of the current density is in clear contrast to the behavior of the VEVs for the field squared and the energy-momentum tensor which suffer surface divergences. For example, the VEV of the field squared diverges as $1 /\left(z-z_{0}\right)^{D-1}$. The feature that the VEV of the current density is finite on the brane could be argued on the base of general arguments. In quantum field theory the ultraviolet divergences in the VEVs of physical observables bilinear in the field are determined by the local geometrical characteristics of the bulk and boundary. On the background of standard AdS geometry with non-compact dimensions the VEV of the current density in the problem under consideration vanishes by the symmetry. The compactification of the part of spatial dimensions to $q$-dimensional torus does not change the local bulk and boundary geometries and, consequently, does not add new divergences to the expectation values compared with the case of trivial topology.

Under the condition (2.35), the contribution of the special mode (2.33) has to be added to the right-hand side of (3.18) (representation (3.7) is not changed). This contribution is obtained from the corresponding part (2.37) in the Hadamard function and is related to the current density for a massless scalar field in $D$-dimensional Minkowski spacetime with the spatial topology $R^{p} \times T^{q}$ by the formula $\left\langle j^{l}\right\rangle_{(\mathrm{R})}=\Omega_{(\mathrm{R})}^{2}(z)\left\langle j^{l}\right\rangle_{R^{p} \times T^{q}}^{(\mathrm{M})}$, where the factor $\Omega_{(\mathrm{R})}^{2}(z)$ is given by the expression (2.36). By using the expression for $\left\langle j^{l}\right\rangle_{R^{p} \times T^{q}}^{(\mathrm{M})}$ from [41], 
one gets

$$
\left\langle j^{l}\right\rangle_{(\mathrm{R})}=\frac{8 e(\nu-1) z_{0}^{2 \nu-2} z^{D-2 \nu}}{(2 \pi)^{(p+3) / 2} a^{D-1} V_{q} L_{l}^{p}} \sum_{n=1}^{\infty} \frac{\sin \left(n \tilde{\alpha}_{l}\right)}{n^{p+1}} \sum_{\mathbf{n}_{q-1}} g_{p / 2+1}\left(n L_{l} k_{(q-1)}\right) .
$$

Recall that, as the necessary condition for the presence of this contribution we have $\nu>1$. In the model with a single compact dimension this gives

$$
\left\langle j^{l}\right\rangle_{(\mathrm{R})}=\frac{4 e(\nu-1) \Gamma(D / 2)}{\pi^{D / 2} a^{D-1} L^{D-1}} z_{0}^{2 \nu-2} z^{D-2 \nu} \sum_{n=1}^{\infty} \frac{\sin \left(n \tilde{\alpha}_{l}\right)}{n^{D-1}} .
$$

Note that in the case $\nu=D / 2$, the current density from the special mode does not depend on $z$.

Let us consider the behavior of the current density in the limit when both the location of the brane and the point of observation are close to the AdS boundary, $z_{0}, z \ll L_{i}$. Under this condition, the arguments of the Bessel functions $J_{\nu}(x)$ and $Y_{\nu}(x)$ in (3.18) are small and we use the corresponding asymptotic expressions. To the leading order, the integral over $\lambda$ is expressed in terms of the Macdonald function and we get the expression

$$
\begin{aligned}
\left\langle j^{l}\right\rangle \approx & \frac{e a^{-D-1} z^{D-2 \nu+2} L_{l}^{-p-2 \nu-2}}{2^{p / 2+\nu-1} \pi^{p / 2+1} \Gamma(\nu+1) V_{q}}\left(z^{2 \nu}-\frac{A_{0}+\nu B_{0}}{A_{0}-\nu B_{0}} z_{0}^{2 \nu}\right)^{2} \\
& \times \sum_{n=1}^{\infty} \frac{\sin \left(n \tilde{\alpha}_{l}\right)}{n^{p+2 \nu+3}} \sum_{\mathbf{n}_{q-1}} g_{p / 2+\nu+2}\left(n L_{l} k_{(q-1)}\right) .
\end{aligned}
$$

The part with the term $z^{2 \nu}$ in the brackets gives the asymptotic for the VEV in the absence of the brane.

The representation (3.18) is also well-suited for the investigation of the asymptotic behavior in the limit of large $L_{l}$ compared with the other length scales of the model. In this limit the argument of the function $g_{\mu}(x)$ in (3.18) is large and we can use the asymptotic expression $g_{\mu}(x) \approx \sqrt{\pi / 2} x^{\mu-1 / 2} e^{-x}$, for $x \gg 1$. The dominant contribution to the integral comes from the region near the lower limit. Let us denote by $k_{(q-1)}^{(0)}$ the lowest value of $k_{(q-1)}, k_{(q-1)}^{(0)}=\min \left(k_{(q-1)}\right)$. For $\left|\tilde{\alpha}_{i}\right|<\pi$ this value is realized by the mode with $n_{i}=0$, $i \neq l$, and we have

$$
k_{(q-1)}^{(0) 2}=\sum_{i=p+1, \neq l}^{D-1} \tilde{\alpha}_{i}^{2} / L_{i}^{2} .
$$

Two cases should be considered separately. For $k_{(q-1)}^{(0)} \neq 0$, in the series over $n$ the leading contribution comes from the $n=1$ term and we get

$$
\begin{aligned}
\left\langle j^{l}\right\rangle \approx & \frac{2 e a^{-D-1} z^{D-2 \nu+2} k_{(q-1)}^{(0)(p+3) / 2+\nu}}{\pi^{(p+1) / 2} \Gamma(\nu+1) V_{q}\left(2 L_{l}\right)^{(p+1) / 2+\nu}} \\
& \times\left(z^{2 \nu}-\frac{A_{0}+\nu B_{0}}{A_{0}-\nu B_{0}} z_{0}^{2 \nu}\right)^{2} \frac{\sin \tilde{\alpha}_{l}}{e^{L_{l} k_{(q-1)}^{(0)}}} .
\end{aligned}
$$


In this case the current density is exponentially small. Note that (3.25) is also obtained from (3.23) in the limit of large $L_{l}$. For $k_{(q-1)}^{(0)}=0$, by using the standard integral involving the Macdonald function [53], for the leading term in the asymptotic expansion one finds the expression

$$
\begin{aligned}
\left\langle j^{l}\right\rangle \approx & \frac{4 e \Gamma(p / 2+\nu+2) a^{-D-1} z^{D-2 \nu+2}}{\pi^{p / 2+1} \Gamma(\nu+1) V_{q} L_{l}^{p+2 \nu+2}} \\
& \times\left(z^{2 \nu}-\frac{A_{0}+\nu B_{0}}{A_{0}-\nu B_{0}} z_{0}^{2 \nu}\right)^{2} \sum_{n=1}^{\infty} \frac{\sin \left(n \tilde{\alpha}_{l}\right)}{n^{p+2 \nu+3}} .
\end{aligned}
$$

Now the decay of the current density with increasing $L_{l}$ is power law for both massless and massive fields. This result for massive fields is in contrast to the corresponding behavior of the current density in Minkowski bulk. In the latter geometry the decay of the current density is exponential with the factor $e^{-m L_{l}}$.

For points outside the brane, $z>z_{0}$, another expression for the current density is obtained from the representation (A.5). After evaluating the integrals, one finds the following result

$$
\begin{aligned}
\left\langle j^{l}\right\rangle= & \left\langle j^{l}\right\rangle_{0}-\frac{4 e a^{-1-D} L_{l}^{-p} z^{D+2}}{(2 \pi)^{p / 2+1} V_{q}} \sum_{n=1}^{\infty} \frac{\sin \left(n \tilde{\alpha}_{l}\right)}{n^{p+1}} \sum_{\mathbf{n}_{q-1}} \int_{k_{(q-1)}}^{\infty} d x \\
& \times x \frac{\bar{I}_{\nu}\left(x z_{0}\right)}{\bar{K}_{\nu}\left(x z_{0}\right)} K_{\nu}^{2}(x z) w_{p / 2+1}\left(n L_{l} \sqrt{x^{2}-k_{(q-1)}^{2}}\right) .
\end{aligned}
$$

with the function

$$
w_{\nu}(x)=x^{\nu} J_{\nu}(x)
$$

In the absence of the bound states, the equivalence of the representations (3.7) and (3.27) for the brane-induced contribution is directly seen by using the formula

$$
\sum_{n_{l}=-\infty}^{+\infty} k_{l} g\left(\left|k_{l}\right|\right)=\frac{2 L_{l}}{\pi} \sum_{n=1}^{\infty} \sin \left(n \tilde{\alpha}_{l}\right) \int_{0}^{\infty} d x x \sin \left(n L_{l} x\right) g(x) .
$$

The latter relation follows from the Poisson's resummation formula (see also [46]).

In what follows, all the graphs are plotted in the $D=4$ model with a single compact dimension of the coordinate length $L$ and with the phase $\tilde{\alpha}$, for a minimally coupled $(\xi=0)$ massless scalar field. For the corresponding value of the parameter $\nu$ one has $\nu=D / 2=2$.

On the left panel of figure 2 we have depicted the current density as a function of the phase $\tilde{\alpha}$ for fixed values of the parameters $z_{0} / L=1, z / z_{0}=1.2$. The graphs are plotted for Dirichlet (D), Neumann (N) and for Robin (with $\beta / a=-1$, the number near the curve) boundary conditions. The dashed curve presents the current density in the same model when the brane is absent. As is seen, depending on the boundary condition, the presence of the brane leads to the increase or decrease of the current density. Note that for the example considered one has $\nu-D / 2=0$ and, in accordance with (2.35), for Neumann boundary condition there is a special mode (2.33) the contribution of which is given by (3.21). This contribution should be added to (3.20). Alternatively, in the numerical evaluation we may 

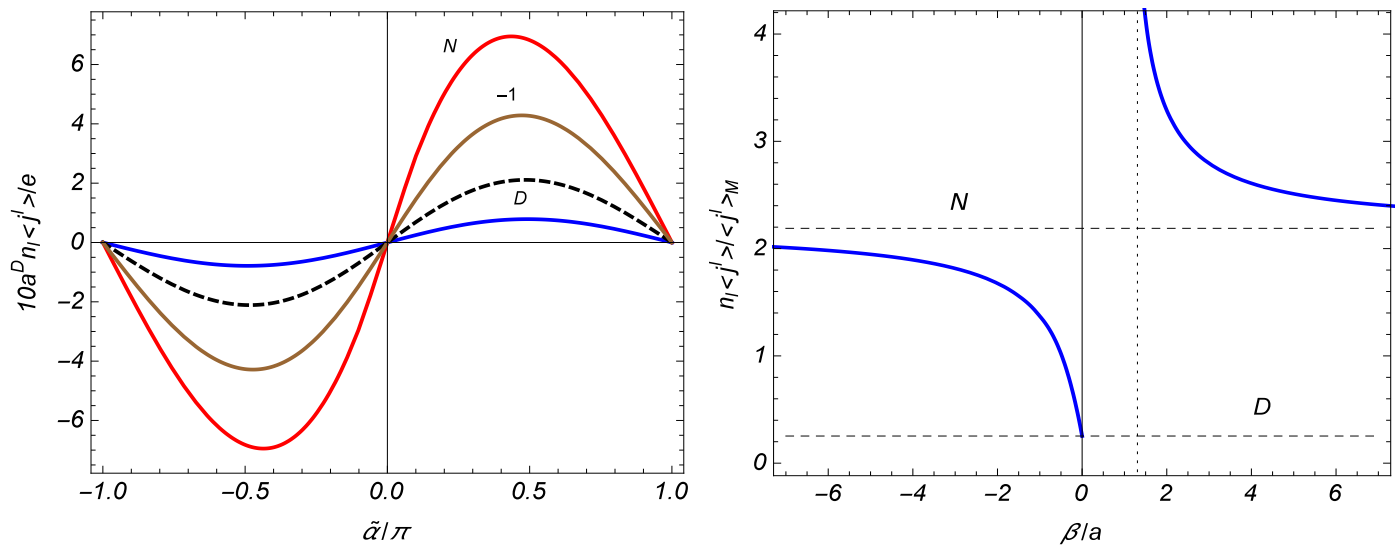

Figure 2. The VEV of the current density as a function of the phase in the periodicity condition (left panel) for $D=4$ AdS space with a single compact dimension and for Dirichlet, Neumann and Robin $(\beta / a=-1)$ boundary conditions. The graphs are plotted for $z_{0} / L=1, z / z_{0}=1.2$. The right panel displays the ratio $n_{l}\left\langle j^{l}\right\rangle /\left\langle j^{l}\right\rangle_{\mathrm{M}}$ as a function of the coefficient in Robin boundary condition for fixed values $\tilde{\alpha}=\pi / 2, z / z_{0}=1.2, z_{0} / L=1$.

use the representation (3.7) which holds in the presence of the special mode as well. We have numerically checked that both these ways of the evaluation give the same result.

The right panel of figure 2 presents the ratio $n_{l}\left\langle j^{l}\right\rangle /\left\langle j^{l}\right\rangle_{\mathrm{M}}$ as a function of the coefficient in Robin boundary condition, measured in units of AdS curvature radius. Here,

$$
\left\langle j^{l}\right\rangle_{\mathrm{M}}=\frac{2 e \Gamma((D+1) / 2)}{\pi^{(D+1) / 2}(a L / z)^{D}} \sum_{n=1}^{\infty} \frac{\sin (\tilde{\alpha} n)}{n^{D}},
$$

is the current density for a massless scalar field in $(D+1)$-dimensional Minkowski spacetime with topology $R^{D-1} \times S^{1}$ and with the length of the compact dimension $a L / z$. Note that the latter is the proper length of the compact dimension in AdS spacetime measured by an observer with a given $z$. The graph is plotted for fixed values $\tilde{\alpha}=\pi / 2, z / z_{0}=1.2$, $z_{0} / L=1$. The vertical dotted curve corresponds to the critical value $\beta_{\mathrm{R}} / a \approx 1.31$. In the region $0<\beta<\beta_{\mathrm{R}}$ the vacuum is unstable. The horizontal dashed curves correspond to Dirichlet and Neumann boundary conditions. As expected, in the limits $\beta \rightarrow 0$ and $\beta \rightarrow \infty$ the results for Dirichlet and Neumann conditions are obtained. For Dirichlet boundary condition the current density takes its minimal value (the minimal absolute value for negative $\tilde{\alpha})$.

In figure 3 we have plotted the ratio $n_{l}\left\langle j^{l}\right\rangle /\left\langle j^{l}\right\rangle_{\mathrm{M}}$ as a function of $z / z_{0}$ for Dirichlet (left panel) and Neumann (right panel) boundary conditions on the brane. The numbers near the curves are the values of $z_{0} / L$. For the phase we have taken the value $\tilde{\alpha}=\pi / 2$. As it has been already mentioned, for Dirichlet boundary condition the current density vanishes on the brane. From the asymptotic analysis given above it follows that on the horizon, $z / z_{0} \rightarrow \infty$, one has $n_{l}\left\langle j^{l}\right\rangle /\left\langle j^{l}\right\rangle_{\mathrm{M}} \rightarrow 1$. This behavior is seen from the graphs.

Figure 4 displays the dependence of $n_{l}\left\langle j^{l}\right\rangle /\left\langle j^{l}\right\rangle_{\mathrm{M}}$ on $z / z_{0}$ in the case of Robin boundary condition for several values of $\beta / a$ (numbers near the curves). For $\beta / a=-2 /(D-1)$ the 

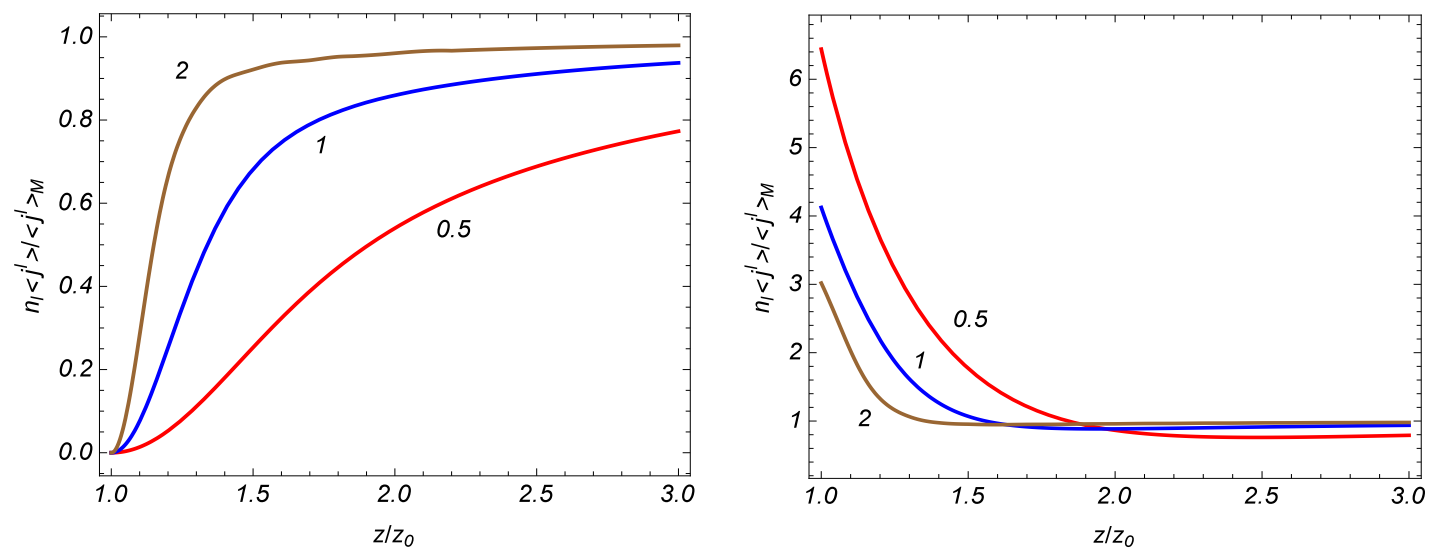

Figure 3. The dependence of the quantity $n_{l}\left\langle j^{l}\right\rangle /\left\langle j^{l}\right\rangle_{\mathrm{M}}$ on $z / z_{0}$ for Dirichlet (left panel) and Neumann (right panel) boundary conditions. The graphs are plotted for fixed values of $z_{0} / L$ (figures near the curves) and for $\tilde{\alpha}=\pi / 2$.

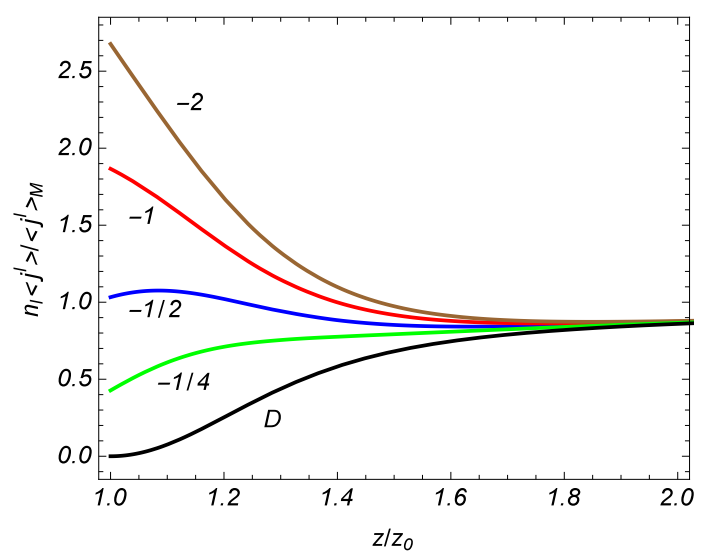

Figure 4. The same as in figure 3 for Robin boundary condition. The graphs are plotted for $z_{0} / L=1$ and the numbers near the curves correspond to the values of $\beta / a$.

normal derivative $\partial_{z}\left(n_{l}\left\langle j^{l}\right\rangle /\left\langle j^{l}\right\rangle_{\mathrm{M}}\right)$ vanishes on the brane. This can also be directly seen from the analytic expression by taking into account that $\left.v \partial_{v} g_{\nu}(u, v)\right|_{v=u}=-2 A_{0} / \pi$.

From the results derived in this section we can obtain the current density in $Z_{2^{-}}$ symmetric braneworld models of the Randall-Sundrum type with a single brane. In the original Randall-Sundrum 1-brane model [54] the universe is realized as a $Z_{2}$-symmetric positive tension brane in 5-dimensional AdS spacetime. In this simplest variant the only contribution to the curvature comes from the negative cosmological constant in the bulk. However, most scenarios motivated from string theories predict the presence of other bulk fields, such as scalar fields. In addition, they predict also small compact dimensions originating from 10D string backgrounds. In a generalized $(D+1)$-dimensional version of the Randall-Sundrum 1-brane model the line element is given by (2.2) with the warp factor $e^{-\left|y-y_{0}\right| / a}$ where $y_{0}$ is the location of the brane. The background geometry contains two 


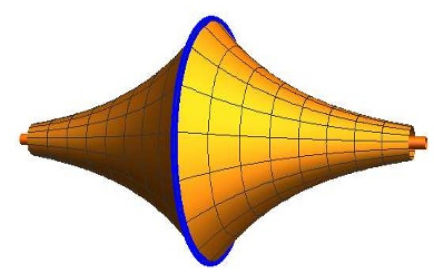

Figure 5. $D=2$ spatial geometry corresponding to the Randall-Sundrum 1-brane model with a compactified dimension.

patches $y>y_{0}$ of the AdS glued by the brane and related by the $Z_{2}$-symmetry identification $y-y_{0} \longleftrightarrow y_{0}-y$. The corresponding spatial geometry in the case $D=2$, embedded into a 3D Euclidean space is depicted in figure 5.

Because of the absolute value sign in the exponent of the warp factor, the Ricci scalar contains a contribution located on the brane,

$$
R=4 D \delta\left(y-y_{0}\right) / a-D(D+1) / a^{2} .
$$

For non-minimally coupled scalar fields, this leads to delta-type terms in the field equation. An additional delta-type term may come from the boundary action of a scalar field of the form $S_{b}=c \int d^{D} x d y \sqrt{|g|} \varphi^{2} \delta\left(y-y_{0}\right)$, where $c$ is the so-called brane mass term. The boundary condition for the mode functions is obtained by integrating the field equation near the brane. In a way similar to that used in [19-21], it can be seen that for fields even under the reflection with respect to the brane (untwisted scalar field) the boundary condition is of the Robin type with

$$
\beta=-1 /(c+2 D \xi / a) .
$$

In particular, for a minimally coupled field with the zero brane mass term the boundary condition is the Neumann one. For fields odd with respect to the reflection (twisted fields) the boundary condition is reduced to the Dirichlet one. Now, in the $Z_{2}$-symmetric model the integration over $y$ in the normalization integral (2.20) goes over the interval $(-\infty,+\infty)$. As a result the square of the normalization coefficient contains an additional factor $1 / 2$ compared to the one we have obtained for the R-region. Hence, the expressions for the VEV of the current density in the generalized Randall-Sundrum 1-brane model with compact dimensions are obtained from those given above in this section with an additional factor $1 / 2$ and with the Robin coefficient (3.32) for untwisted fields and with $\beta=0$ for twisted fields.

\subsection{L-region}

Now we turn to the current density in the L-region. By using the expression (2.45) for the Hadamard function, the current density in this region is decomposed as (3.2) with the 
brane-induced part

$$
\left\langle j^{l}\right\rangle_{b}=-\frac{e C_{p} z^{D+2}}{2^{p-1} a^{D+1} V_{q}} \sum_{\mathbf{n}_{q}} k_{l} \int_{k_{(q)}}^{\infty} d x x\left(x^{2}-k_{(q)}^{2}\right)^{\frac{p-1}{2}} \frac{\bar{K}_{\nu}\left(z_{0} x\right)}{\bar{I}_{\nu}\left(z_{0} x\right)} I_{\nu}^{2}(z x) .
$$

Under the condition $\eta<k_{(q)}^{(0)}$, this representation is valid in the presence of the mode with $\lambda=i \eta$, where $\eta$ is the zero of the function $\bar{I}_{\nu}\left(z_{0} \eta\right)$, and also in the presence of the special mode (2.48). For large values of AdS radius $a$, in a way similar to that for the R-region, we can see the limiting transition of the expression (3.33) to the corresponding formula for a plate in Minkowski bulk.

For a conformally coupled massless field, the expression of the total current density takes the form

$$
\begin{aligned}
\left\langle j^{l}\right\rangle= & (z / a)^{D+1}\left\{\left\langle j^{l}\right\rangle_{R^{p+1} \times T^{q}}^{(\mathrm{M})}-\frac{e C_{p}}{2^{p} V_{q}} \sum_{\mathbf{n}_{q}} k_{l} \int_{k_{(q)}}^{\infty} d x\right. \\
& \left.\times\left(x^{2}-k_{(q)}^{2}\right)^{\frac{p-1}{2}}\left[e^{-2 z x}+\frac{4 \sinh ^{2}(z x)}{\frac{1-\beta_{\mathrm{M}}^{-} x}{1+\beta_{\mathrm{M}}^{-}} e^{2 z_{0} x}-1}\right]\right\},
\end{aligned}
$$

with $\beta_{\mathrm{M}}^{-}$defined by (3.11). Here, the first term in the figure braces and the part with the first term in the square brackets come from $\left\langle j^{l}\right\rangle_{0}$. The expression on the right of (3.34), divided by the conformal factor $(z / a)^{D+1}$, coincides with the current density in the region between two plates on Minkowski bulk with Dirichlet boundary condition on the left plate and Robin condition (2.8), with $\beta \rightarrow \beta_{\mathrm{M}}^{-}$, on the right one (see [46] for the problem with Robin boundary conditions on both plates). The fact that the problem with a single brane in AdS bulk in the L-region is conformaly related to the problem with two plates in Minkowski bulk is a consequence of the boundary condition we have imposed on the AdS boundary.

The asymptotic behavior of the VEV near the AdS boundary, $z \rightarrow 0$, is directly obtained from (3.33), by using the expression of the modified Bessel function for small arguments. To the leading order we get

$$
\begin{aligned}
\left\langle j^{l}\right\rangle_{b} \approx & -\frac{2^{1-2 \nu-p} e C_{p} z^{D+2 \nu+2}}{a^{D+1} V_{q} \Gamma^{2}(\nu+1)} \sum_{\mathbf{n}_{q}} k_{l} k_{(q)}^{2 \nu+p+1} \\
& \times \int_{1}^{\infty} d x x^{2 \nu+1}\left(x^{2}-1\right)^{(p-1) / 2} \frac{\bar{K}_{\nu}\left(z_{0} k_{(q)} x\right)}{\bar{I}_{\nu}\left(z_{0} k_{(q)} x\right)},
\end{aligned}
$$

and the brane-induced contribution vanishes as $z^{D+2 \nu+2}$. Recall that near the AdS boundary the part $\left\langle j^{l}\right\rangle_{0}$ in the VEV of the current density behaves in a similar way and, hence, on the AdS boundary the ratio of the brane-induced and boundary-free contributions tend to a finite limiting value.

In the limit when the brane tends to the AdS horizon, $z_{0} \rightarrow \infty$, the argument $z_{0} x$ of the modified Bessel functions is large. By using the corresponding asymptotics and by taking into account that the dominant contribution to the integral in (3.33) comes from 
the region near the lower limit, we can see that the leading order term is given by

$$
\left\langle j^{l}\right\rangle_{b} \approx \frac{\left(1-2 \delta_{0 B_{0}}\right) e \tilde{\alpha}_{l} z^{D+2} e^{-2 z_{0} k_{(q)}^{(0)}}}{2^{p} \pi^{(p-1) / 2} a^{D+1} V_{q} L_{l} z_{0}^{(p+1) / 2}} k_{(q)}^{(0)(p+1) / 2} I_{\nu}^{2}\left(z k_{(q)}^{(0)}\right) .
$$

Hence, for a fixed value of $z$, when the brane location tends to the AdS horizon, the brane-induced contribution is exponentially suppressed.

If the length of the $l$ th dimension is much smaller than the lengths of the remaining compact dimensions, $L_{l} \ll L_{i}$, in a way similar to that for the R-region, we can see that, to the leading order, the brane-induced contribution coincides with the corresponding quantity in the model with a single compact dimension of the length $L_{l}$. The expression for the latter is obtained from the right-hand side of (3.15) by the replacements $I_{\nu} \rightleftarrows K_{\nu}$. If in addition $L_{l} \ll z$, the corresponding asymptotic expression is given by the right-hand side of (3.16) with $z_{0}-z$ instead of $z-z_{0}$, and the brane-induced contribution is concentrated near the brane in the region $z_{0}-z \lesssim L_{l}$.

The representation (3.33) is not well adapted for the investigation of the asymptotic near the brane. A more suitable representation is obtained by using the formula (A.6) for the Hadamard function:

$$
\begin{aligned}
\left\langle j^{l}\right\rangle= & \frac{16 e a^{-1-D} z^{D+2}}{(2 \pi)^{p / 2+1} V_{q} L_{l}^{p} z_{0}^{2}} \sum_{n=1}^{\infty} \frac{\sin \left(n \tilde{\alpha}_{l}\right)}{n^{p+1}} \sum_{\mathbf{n}_{q-1}} \sum_{i} \gamma_{i} \\
& \times T_{\nu}\left(\gamma_{i}\right) J_{\nu}^{2}\left(\gamma_{i} z / z_{0}\right) g_{p / 2+1}\left(n L_{l} \sqrt{\gamma_{i}^{2} / z_{0}^{2}+k_{(q-1)}^{2}}\right) .
\end{aligned}
$$

Unlike to the representation (3.33), in the presence of a bound state, its contribution must be additionally added to (3.37). The latter is obtained from the right-hand side of (3.37) by the replacement $\gamma_{i} \rightarrow i \eta z_{0}$ and omitting the summation over $i$. The corresponding representation is valid under the condition $\eta<k_{(q-1)}^{(0)}$. From (3.37) we conclude that the VEV of the current density is finite on the brane. Similar to the VEV in the R-region, the current density and its normal derivative vanish on the brane for Dirichlet boundary condition. Another representation is obtained from (3.27) by the replacements $I_{\nu} \rightleftarrows K_{\nu}$ and with the replacement $\beta \rightarrow-\beta$ in the notations with overbars.

For the value of the Robin coefficient (2.47) there is a special mode (2.48). As we have seen above, the corresponding contribution to the Hadamard function is expressed in terms of the Hadamard function for a massless scalar field in $D$-dimensional Minkowski spacetime with the spatial topology $R^{p} \times T^{q}$. By using this relation, for the contribution of the special mode to the current density in the L-region we find

$$
\left\langle j^{l}\right\rangle_{(\mathrm{L})}=\frac{8 e(\nu+1) z_{0}^{-2 \nu-2} z^{D+2 \nu}}{(2 \pi)^{(p+3) / 2} a^{D-1} V_{q} L_{l}^{p}} \sum_{n=1}^{\infty} \frac{\sin \left(n \tilde{\alpha}_{l}\right)}{n^{p+1}} \sum_{\mathbf{n}_{q-1}} g_{p / 2+1}\left(n L_{l} k_{(q-1)}\right) .
$$

In the case of a single compact dimension this simplifies to

$$
\left\langle j^{l}\right\rangle_{(\mathrm{L})}=\frac{4 e(\nu+1) \Gamma(D / 2) z^{D+2 \nu}}{\pi^{D / 2} a^{D-1} L^{D-1} z_{0}^{2 \nu+2}} \sum_{n=1}^{\infty} \frac{\sin \left(n \tilde{\alpha}_{l}\right)}{n^{D-1}} .
$$


Under the condition (2.47), the contribution (3.38) should be added to the right-hand side of (3.37).

For the investigation of the asymptotic behavior for the contribution of the modes with $\lambda=\gamma_{n} / a$ in the limit of large values of $L_{l}$, compared with the other length scales, it is convenient to use the representation (3.37). By using the asymptotic expression of the function $g_{\mu}(x)$ for large values of the argument, we can see that the dominant contribution comes from the lowest mode with $i=1$ and to the leading order one finds

$$
\left\langle j^{l}\right\rangle=\frac{8 e z^{D+2} \gamma_{1} T_{\nu}\left(\gamma_{1}\right) \sin \tilde{\alpha}_{l}}{(2 \pi)^{(p+1) / 2} V_{q} L_{l}^{p} z_{0}^{2} a^{D+1}} \frac{x^{(p+1) / 2}}{e^{-x}} J_{\nu}^{2}\left(\gamma_{1} z / z_{0}\right),
$$

with $x=L_{l} \sqrt{\gamma_{1}^{2} / z_{0}^{2}+k_{(q-1)}^{(0) 2}}$. Hence, unlike to the R-region, the decay of the current density is exponential for both cases $k_{(q-1)}^{(0)}=0$ and $k_{(q-1)}^{(0)} \neq 0$. Under the condition (2.47) one has the additional contribution (3.38) from the mode with $\lambda=0$. For $k_{(q-1)}^{(0)} \neq 0$, in the limit of large $L_{l}$, this contribution decays exponentially, as $e^{-L_{l} k_{(q-1)}^{(0)}}$. In the case $k_{(q-1)}^{(0)}=0$ the decay is power law, like $1 / L_{l}^{p+1}$. In both cases the contribution of the special mode dominates in the total VEV.

Now let us consider the asymptotic of the current density (3.37) when the brane is close to AdS boundary, $z_{0} \ll L_{i}$. The dominant contribution comes from large values of $\left|n_{j}\right|, j \neq l$, and we can replace the summation over $\mathbf{n}_{q-1}$ by the integration in accordance with (3.14). After the integration over $k_{(q-1)}$ we get

$$
\left\langle j^{l}\right\rangle \approx \frac{16 e a^{-1-D} z^{D+2}}{(2 \pi)^{D / 2} L_{l}^{D-1} z_{0}^{2}} \sum_{n=1}^{\infty} \frac{\sin \left(n \tilde{\alpha}_{l}\right)}{n^{D-1}} \sum_{i} \gamma_{i} T_{\nu}\left(\gamma_{i}\right) J_{\nu}^{2}\left(\gamma_{i} z / z_{0}\right) g_{D / 2}\left(n L_{l} \gamma_{i} / z_{0}\right) .
$$

The right-hand side presents the brane-induced contribution in the model with a single compact dimension of the length $L_{l}$. In the limit under consideration the argument of the function $g_{D / 2}(x)$ is large. By using the corresponding asymptotic expression, we see that the main contribution comes from the term with $n=1, i=1$ with the result

$$
\left\langle j^{l}\right\rangle \approx \frac{8 e a^{-1-D} z^{D+2} \sin \tilde{\alpha}_{l}}{(2 \pi)^{(D-1) / 2} L_{l}^{D+1}} \frac{\left(\gamma_{1} L_{l} / z_{0}\right)^{(D+3) / 2}}{\gamma_{1} e^{\gamma_{1} L_{l} / z_{0}}} T_{\nu}\left(\gamma_{1}\right) J_{\nu}^{2}\left(\gamma_{1} z / z_{0}\right) .
$$

For the Robin boundary condition with (2.47), the asymptotic for the contribution from the special mode is directly obtained from (3.38). This contribution behaves as $\left(z_{0} / L_{l}\right)^{D-2}\left(z / z_{0}\right)^{D+2 \nu}$ and the corresponding decay, as a function of $z_{0}$ (for a fixed $z / z_{0}$ ) is power law.

The left panel of figure 6 displays the vacuum current density in the L-region for Dirichlet, Neumann and Robin (with $\beta / a=-1 / 2$ ) boundary conditions as a function of the phase in the quasiperiodicity condition along the compact dimensions. Recall that in the numerical evaluations we consider a $D=4$ minimally coupled massless field. In this case $\nu=2$ and the modes with purely imaginary $\lambda$ and the special mode with $\lambda=0$ are absent for $a / \beta<4$. The graphs are plotted for $z_{0} / L=1, z / z_{0}=0.8$. The dashed curve corresponds to the current density in the absence of the brane. The right panel of 

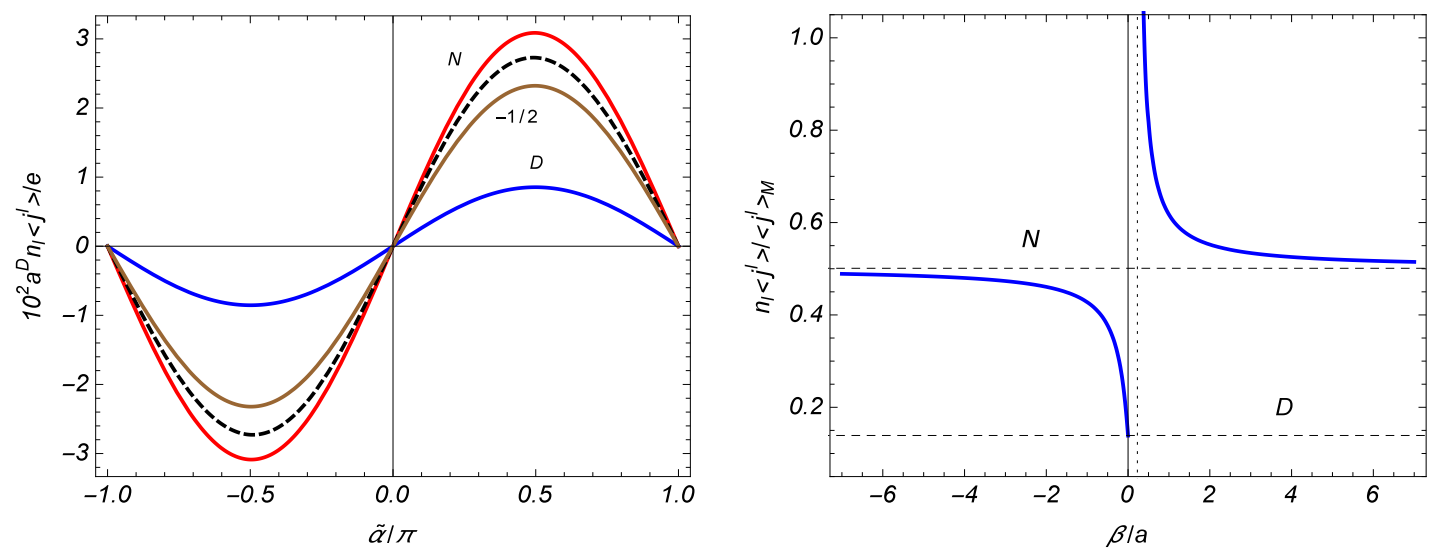

Figure 6. The current density in the L-region as a function of $\tilde{\alpha}$ for Dirichlet, Neumann and Robin (with $\beta / a=-1 / 2$ ) boundary conditions (left panel) and as a function of the coefficient in Robin boundary condition (right panel). The graphs are plotted for $z_{0} / L=1, z / z_{0}=0.8$. On the left panel the dashed curve corresponds to the current density in the geometry without the brane. The vertical dotted line on the right panel corresponds to the critical value $\beta_{c} / a \approx 0.228$.

figure 6 presents the dependence of the ratio $n_{l}\left\langle j^{l}\right\rangle /\left\langle j^{l}\right\rangle_{\mathrm{M}}$ on $\beta / a$. The horizontal dashed lines correspond to Dirichlet and Neumann boundary conditions. The vertical dotted line corresponds to the critical value $\beta_{c} / a \approx 0.228$. In the region $0<\beta<\beta_{c}$ the vacuum is unstable.

In figure 7 , for $\tilde{\alpha}=\pi / 2$, we show the dependence of the ratio $n_{l}\left\langle j^{l}\right\rangle /\left\langle j^{l}\right\rangle_{\mathrm{M}}$ on $z / z_{0}$ for Dirichlet (left panel) and Neumann (right panel) boundary conditions for separate values of $z_{0} / L$ (numbers near the curves). For Dirichlet condition the current density vanishes on the AdS boundary and on the brane. As it follows from the asymptotic (3.35), near the AdS boundary the charge flux density $n_{l}\left\langle j^{l}\right\rangle$ behaves as $z^{D+2 \nu+1}$. For the Minkowskian VEV with the length of the compact dimension $a L / z$, equal to the proper length on the AdS bulk, one has $\left\langle j^{l}\right\rangle_{\mathrm{M}} \propto z^{D+1}$. Hence, the ratio plotted in figuire 7 vanishes on the AdS boundary as $z^{2 \nu}$. Similar graphs in the case of Robin boundary condition are presented in figure 8 for several values of $\beta / a$ (numbers near the curves). Note that for all the examples in the L-region there are no modes with purely imaginary $\lambda$.

\section{Conclusion}

We have studied the effects induced by a brane, parallel to the AdS boundary, on the VEV of the current density for a massive charged scalar field with an arbitrary curvature coupling parameter. The background geometry under consideration is obtained from the $(D+1)$-dimensional AdS one, described in Poincaré coordinates, by a toroidal compactification of a part of spatial dimensions. Along compact dimensions the field operator obeys quasiperiodicity conditions with general constant phases. We have also assumed the presence of a constant gauge field. By the gauge transformation the problem is reduced to the one with a zero gauge field. In the new gauge the phases in the periodicity conditions are 

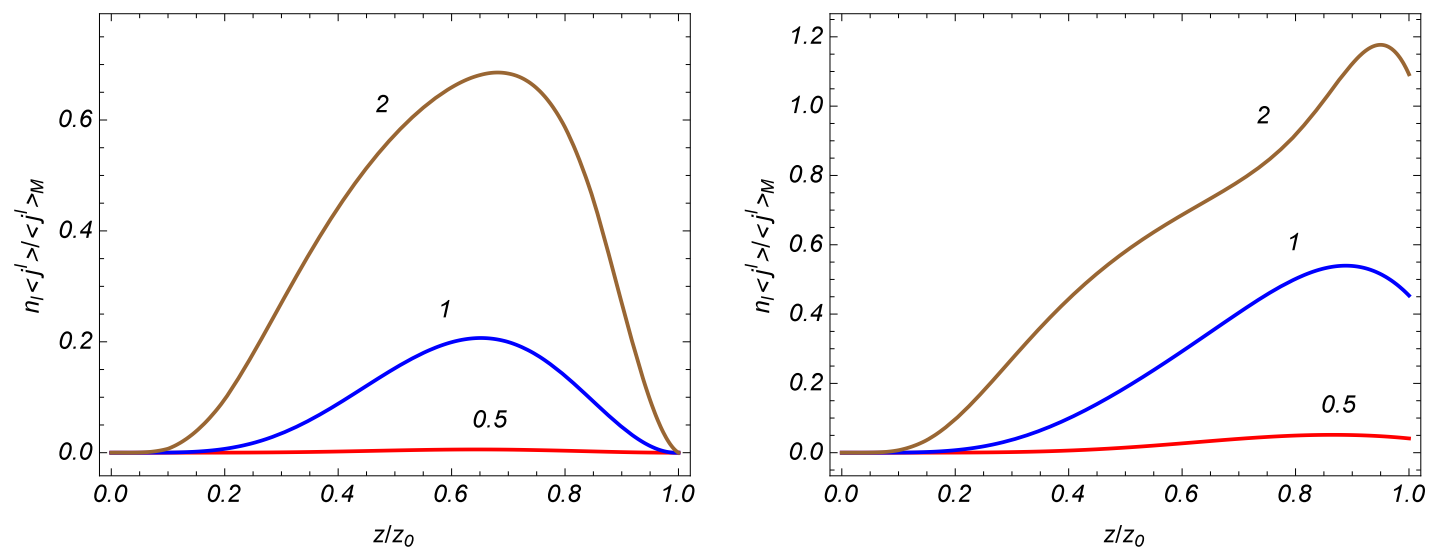

Figure 7. The ratio $n_{l}\left\langle j^{l}\right\rangle /\left\langle j^{l}\right\rangle_{\mathrm{M}}$ versus $z / z_{0}$ for Dirichlet (left panel) and Neumann (right panel) boundary conditions. The figures near the curves correspond to the values of $z_{0} / L$ and for the phase we have taken $\tilde{\alpha}=\pi / 2$.

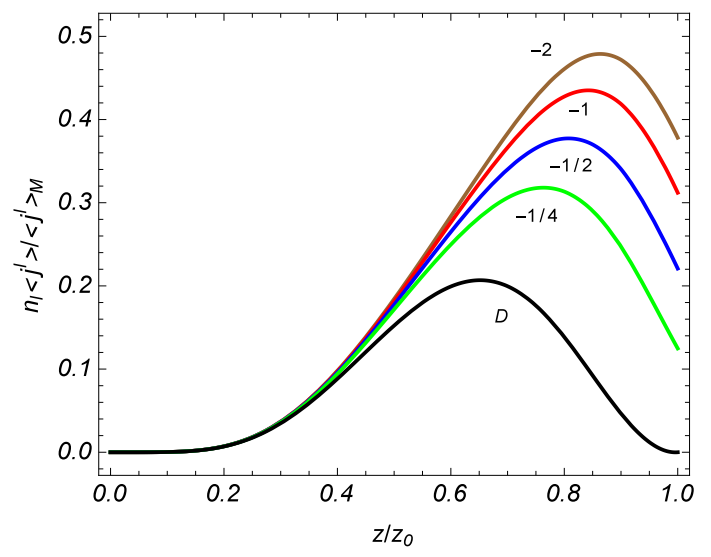

Figure 8. The same as in figure 6 for the fixed value $z / z_{0}=1$ in the case of Robin boundary condition. The numbers near the curves correspond to the values of $\beta / a$.

shifted by an amount determined by the ratio of the magnetic flux, enclosed by a compact dimension, to the flux quantum. On the brane and on the AdS boundary the field operator is constrained by Robin and Dirichlet boundary conditions, respectively. We consider a non-interacting quantum field and the Hadamard two-point function contains all the information about the properties of the vacuum state. Though the background geometry is homogeneous, the extrinsic curvature tensor of the brane is nonzero and, as a consequence of this, the regions on the right (R-region) and on the left (L-region) of the brane are not physically equivalent.

In the R-region the spectrum of the quantum number $\lambda$ is continuous and the mode functions obeying the boundary condition on the brane have the form (2.12) with the radial function (2.16). In addition to these modes, under the condition $a / \beta>\nu-D / 2$, one can have a bound state with the mode function (2.26) and with the eigenvalue for $\eta$ being the 
root of the equation (2.28). The root should be constrained by $\eta<k_{(q)}^{(0)}$, where $k_{(q)}^{(0)}$ is defined as $(2.27)$. For the modes with $\eta>k_{(q)}^{(0)}$ the energy becomes imaginary and they lead to the instability of the Poincaré vacuum state. In addition to the parameters $\nu$ and $k_{(q)}^{(0)}$, the stability of the vacuum depends on the location of the brane. Depending on the value of the Robin coefficient, the approaching of the brane to AdS boundary can lead to the instability. For the value of the Robin coefficient given by (2.35) there is a special mode (2.33) for which $\lambda=0$. For a minimally coupled massless scalar field the special value of the Robin coefficient corresponds to Neumann boundary condition and the mode function does not depend on the radial coordinate $z$. This is an analog of the graviton zero mode in Randall-Sundrum 1-brane model. The Hadamard function in the R-region is given by the expression (2.25) with the second term in the right-hand side being the brane-induced contribution. We have shown that this expression holds in the presence of bound states as well. Alternative representations for the Hadamard function in the R-region, (A.3) and (A.5), are derived in appendix, by using the summation formula (A.1).

In the L-region the spectrum for $\lambda$ is discrete and its eigenvalues are roots of the equation (2.40). The corresponding expression for the Hadamard function contains series over these roots. Another expression, in which the explicit knowledge of the eigenvalues for $\lambda$ is not required is obtained by making use of the generalized Abel-Plana formula (2.44). This allows to extract manifestly the brane-induced contribution, given by the second term in the right-hand side of (2.45). For $a / \beta>D / 2+\nu$ there is a mode with purely imaginary $\lambda=i \eta$ which is the root of the equation (2.46) with $x=\eta z_{0}$. For the stability of the vacuum state one needs to have $\eta<k_{(q)}^{(0)}$. The stability condition depends also on the position of the brane. The shift of the brane to the direction of the AdS boundary gives rise to the instability in the initially stable vacuum state. An alternative expression for the Hadamard function in the L-region is given by (A.6).

In both the R- and L-regions the VEV of the current density is decomposed into the boundary-free and brane-induced contributions. For both these contributions, the component of the current density along the $l$ th compact dimension is an odd periodic function of the phase $\tilde{\alpha}_{l}$ and an even periodic function of the phases $\tilde{\alpha}_{i}, i \neq l$, with the period equal to $2 \pi$. In the R-region the brane-induced contribution is given by (3.7). We have checked that for large values of the AdS curvature radius the leading term in the corresponding asymptotic expansion coincides with the boundary-induced part of the current density in Minkowski spacetime with topology $R^{p+1} \times T^{q}$ in the presence of a single Robin plate. In the case of a conformally coupled massless field the current density coincides, up to the conformal factor $(z / a)^{D+1}$, with the corresponding quantity in Minkowski bulk with the plate at $z=z_{0}$ on which the field obeys the Robin boundary condition with the coefficient $\beta_{\mathrm{M}}^{(+)}$ given by (3.11). For points near the AdS horizon ( $z$ is large compared with the other length scales), the brane-induced contribution is suppressed by the factor $e^{-2 z k_{(q)}^{(0)}}$. In the same limit the boundary-free part behaves as $(z / a)^{D+1}$ and it dominates in the total VEV. In the limit when the brane approaches to the AdS boundary, for fixed values of $z$ and $L_{i}$, the brane-induced contribution tends to zero like $z_{0}^{2 \nu}$. For the investigation of the near brane asymptotic of the vacuum current it is more convenient to use the representation (3.18). In 
the presence of a bound state its contribution should be added separately to the right-hand side. For Robin boundary condition with (2.35) an additional contribution, given by (3.21), comes from the special mode (2.33). An important conclusion which follows from the representation (3.18) is that the current density is finite on the brane. This behavior is in clear contrast with that for the VEVs of the field squared and of the energy-momentum tensor which diverge on the brane. For Dirichlet boundary condition the current density and its normal derivative vanish for points on the brane. The asymptotic behavior of the current density along $l$ th compact dimension for large values of the corresponding length $L_{l}$ crucially depends whether the parameter $k_{(q-1)}^{(0)}$, defined by $(3.24)$, is zero or not. For $k_{(q-1)}^{(0)} \neq 0$ the current density decays exponentially like $e^{-L_{l} k_{(q-1)}^{(0)}}$. In the case $k_{(q-1)}^{(0)}=0$ the decay is power law, as $1 / L_{l}^{p+2 \nu+2}$, for both massless and massive fields. This behavior for massive fields is essentially different from that for Minkowski bulk where the current is suppressed exponentially, by the factor $e^{-m L_{l}}$. The expression for the current density in the generalized Randall-Sundrum 1-brane model with compact dimensions is obtained from the formulas in section 3 with an additional factor $1 / 2$ and with the Robin coefficient (3.32) for untwisted fields and with $\beta=0$ for twisted fields.

The current density in the L-region is given by the expression (3.33). For a conformally coupled massless field, this expression is reduced to the one in Minkowski spacetime with two parallel plates, multiplied by the conformal factor $(z / a)^{D+1}$, with Dirichlet boundary condition on the left plate and Robin condition on the right one. On the AdS boundary the brane-induced contribution vanishes as $z^{D+2 \nu+2}$. For a fixed observation point, when the location of the brane tends to the AdS horizon, the brane-induced effects are suppressed by the factor $e^{-2 z_{0} k_{(q)}^{(0)}}$. Similar behavior is exhibited by the boundary-free part. From an alternative representation (3.37) it follows that the current density on the brane is finite and vanishes for Dirichlet boundary condition. For large values of the length $L_{l}$, unlike to the R-region, the decay of the current density in the L-region is exponential for both cases $k_{(q-1)}^{(0)}=0$ and $k_{(q-1)}^{(0)} \neq 0$. This feature is related to the discreteness of the spectrum for $\lambda$. For the value (2.47) of the Robin coefficient the contribution (3.39) from the special mode has to be added to the right-hand side of (3.37). For large values of $L_{l}$, this contribution dominates in the VEV of the total current density. In particular, in the model with a single compact dimension its decay, as a function of $L_{l}$, is power law, as $1 / L_{l}^{D-1}$. For the brane location near the AdS boundary, the asymptotic of the current density is given by (3.42) and it is suppressed by the factor $e^{-\gamma_{1} L_{l} / z_{0}}$. In the presence of the special mode, for a given value of $z / z_{0}$ (fixed distance from the brane), the corresponding contribution to the current density behaves as $\left(z_{0} / L_{l}\right)^{D-2}$ and, hence, it dominates in the total VEV.

The numerical results have been presented for the $D=4$ model with a single compact dimension and for a minimally coupled massless scalar field. These results show that, depending on the value of the Robin coefficient, the presence of the brane can either increase or decrease the current density. In particular, in the example considered, the modulus of the current density takes its minimal value for Dirichlet boundary condition. 


\section{Acknowledgments}

This work has been supported in part by the State Committee of Science of the Ministry of Education and Science of the Republic of Armenia. A. A. S. gratefully acknowledges the hospitality of the INFN, Laboratori Nazionali di Frascati (Frascati, Italy), where part of this work was done.

\section{A Other representations of the two-point function}

Here we provide representations for the Hadamard function convenient in the investigation of near brane asymptoics for the VEV of the current density. First we consider the Rregion. In the representation (2.22) we separate the series over $n_{l}$ and for the summation use the Abel-Plana type formula [42, 43, 64]

$$
\begin{aligned}
\frac{2 \pi}{L_{l}} \sum_{n_{l}=-\infty}^{\infty} g\left(k_{l}\right) f\left(\left|k_{l}\right|\right)= & \int_{0}^{\infty} d u[g(u)+g(-u)] f(u) \\
& +i \int_{0}^{\infty} d u[f(i u)-f(-i u)] \sum_{s= \pm 1} \frac{g(i s u)}{e^{u L_{l}+i s \tilde{\alpha}_{l}}-1}
\end{aligned}
$$

with $k_{l}$ defined in (2.15) (formula (A.1) is reduced to the standard Abel-Plana formula in the special case $\left.g(x)=1, \tilde{\alpha}_{l}=0\right)$. After the application (A.1) with $g\left(k_{l}\right)=e^{i k_{l} \Delta x^{l}}$, the Hadamard function is presented in the form

$$
G\left(x, x^{\prime}\right)=G_{R^{p+2} \times T^{q-1}}\left(x, x^{\prime}\right)+G_{l}\left(x, x^{\prime}\right) .
$$

Here the part $G_{R^{p+2} \times T^{q-1}}\left(x, x^{\prime}\right)$ comes from the first term in the right-hand side of (A.1) and is the Hadamard function for the geometry with a single brane in $(D+1)$ dimensional AdS spacetime with spatial topology $R^{p+2} \times T^{q-1}$ for which the $l$ th dimension is decompactified. The lengths of the remaining compact dimensions are the same: $\left(L_{p+1}, \ldots, L_{l-1}, L_{l+1}, \ldots, L_{D-1}\right)$. The second term on the right of (A.2) is induced by the compactification of the $l$ th dimension and is given by the expression

$$
\begin{aligned}
G_{l}\left(x, x^{\prime}\right)= & \frac{4\left(z z^{\prime}\right)^{D / 2} L_{l}}{(2 \pi)^{p+1} a^{D-1} V_{q}} \sum_{n=1}^{\infty} \sum_{\mathbf{n}_{q-1}} \int d \mathbf{k}_{p} e^{i k_{r} \Delta x^{r}} \int_{0}^{\infty} d \lambda \lambda \\
& \times \frac{g_{\nu}\left(\lambda z_{0}, \lambda z\right) g_{\nu}\left(\lambda z_{0}, \lambda z^{\prime}\right)}{\bar{J}_{\nu}^{2}\left(\lambda z_{0}\right)+\bar{Y}_{\nu}^{2}\left(\lambda z_{0}\right)} \int_{0}^{\infty} d w \cosh (w \Delta t) \\
& \times\left.\frac{e^{-n u L_{l}}}{u} \cosh \left(u \Delta x^{l}+i n \tilde{\alpha}_{l}\right)\right|_{u=\sqrt{w^{2}+\lambda^{2}+k^{(l) 2}}}
\end{aligned}
$$

where $\mathbf{n}_{q-1}=\left(n_{p+1}, \ldots, n_{l-1}, n_{l+1}, \ldots, n_{D-1}\right), k^{(l) 2}=k^{2}-k_{l}^{2}$, and the summation over $r$ in the exponent goes over $r=1, \ldots, D-1, r \neq l$. In deriving this result, we have used the relation

$$
\sum_{s= \pm 1} \frac{e^{-s u \Delta x^{l}}}{e^{u L_{l}+i s \tilde{\alpha}_{l}}-1}=2 \sum_{n=1}^{\infty} e^{-n u L_{l}} \cosh \left(u \Delta x^{l}+i n \tilde{\alpha}_{l}\right) .
$$


Note that the part $G_{R^{p+1} \times T^{q-1}}\left(x, x^{\prime}\right)$ does not contribute to the VEV of the current density along $l$ th dimension. The expression (A.3) gives the contribution to the Hadamard function from the modes with continuous $\lambda$. In the presence of a bound state, a similar representation can be found for the corresponding contribution.

Another useful representation for the Hadamard function in the R-region is obtained by using the identity (2.23) for the integrand in (A.3). The part in $G_{l}\left(x, x^{\prime}\right)$ coming from the first term in the right-hand side of (2.23) gives the corresponding function in the boundaryfree AdS spacetime (the brane is absent), denoted here by $G_{0 l}\left(x, x^{\prime}\right)$. In the part induced by the brane, coming from the last term in (2.23), we rotate the integration contour by the angle $\pi / 2$ for the term with $j=1$ and by the angle $-\pi / 2$ for the term with $j=2$. In this way we get

$$
\begin{aligned}
G_{l}\left(x, x^{\prime}\right)= & G_{0 l}\left(x, x^{\prime}\right)-\frac{a^{1-D} L_{l}\left(z z^{\prime}\right)^{D / 2}}{2^{p-1} \pi^{p+2} V_{q}} \sum_{n=1}^{\infty} \sum_{\mathbf{n}_{q-1}} \int d \mathbf{k}_{p} e^{i k_{r} \Delta x^{r}} \\
& \times \int_{0}^{\infty} d w \cosh (w \Delta t) \int_{0}^{\infty} d u \sum_{s= \pm 1} \cos \left(u \Delta x^{l}-s n \tilde{\alpha}_{l}\right) e^{i s n u L_{l}} \\
& \times\left.\frac{\bar{I}_{\nu}\left(\lambda z_{0}\right)}{\bar{K}_{\nu}\left(\lambda z_{0}\right)} K_{\nu}(\lambda z) K_{\nu}\left(\lambda z^{\prime}\right)\right|_{\lambda=\sqrt{w^{2}+u^{2}+k^{(l) 2}}}
\end{aligned}
$$

Now, we consider the L-region. The corresponding expression for the Hadamard function is given by (2.43). In a way similar to that for the R-region, applying the formula (A.1), for the part induced by the compactification of the $l$ th dimension we find the following representation

$$
\begin{aligned}
G_{l}\left(x, x^{\prime}\right)= & \frac{8\left(z z^{\prime}\right)^{D / 2} L_{l}}{(2 \pi)^{p+1} a^{D-1} V_{q} z_{0}^{2}} \sum_{n=1}^{\infty} \sum_{\mathbf{n}_{q-1}} \int d \mathbf{k}_{p} e^{i k_{r} \Delta x^{r}} \sum_{n} \gamma_{n} \\
& \times T_{\nu}\left(\gamma_{n}\right) J_{\nu}\left(\gamma_{n} z / z_{0}\right) J_{\nu}\left(\gamma_{n} z^{\prime} / z_{0}\right) \int_{0}^{\infty} d w \cosh (w \Delta t) \\
& \times\left.\frac{e^{-n u L_{l}}}{u} \cosh \left(u \Delta x^{l}+i n \tilde{\alpha}_{l}\right)\right|_{u=\sqrt{w^{2}+\gamma_{n}^{2} / z_{0}^{2}+k^{(l) 2}}} .
\end{aligned}
$$

In this expression, for the summation over $n$ we can use the formula (2.44). The part coming from the first term in the right-hand side of (2.44) corresponds to the boundary free contribution and, as a result, we obtain the decomposition similar to (A.5) where now the brane-induced contribution is obtained from that in (A.5) by the replacements $I_{\nu}(x) \rightleftarrows$ $K_{\nu}(x)$.

Open Access. This article is distributed under the terms of the Creative Commons Attribution License (CC-BY 4.0), which permits any use, distribution and reproduction in any medium, provided the original author(s) and source are credited.

\section{References}

[1] J.B. Griffiths and J. Podolský, Exact Space-Times in Einstein's General Relativity, Cambridge University Press, Cambridge U.K. (2009). 
[2] G.W. Gibbons, Anti-de-Sitter spacetime and its uses, arXiv:1110.1206 [INSPIRE].

[3] C.G. Callan Jr. and F. Wilczek, Infrared behavior at negative curvature, Nucl. Phys. B 340 (1990) 366 [InSPIRE].

[4] J.M. Maldacena, The Large- $N$ limit of superconformal field theories and supergravity, Int. J. Theor. Phys. 38 (1999) 1113 [hep-th/9711200] [INSPIRE].

[5] E. Witten, Anti-de Sitter space and holography, Adv. Theor. Math. Phys. 2 (1998) 253 [hep-th/9802150] [INSPIRE].

[6] S.S. Gubser, I.R. Klebanov and A.M. Polyakov, Gauge theory correlators from noncritical string theory, Phys. Lett. B 428 (1998) 105 [hep-th/9802109] [INSPIRE].

[7] O. Aharony, S.S. Gubser, J.M. Maldacena, H. Ooguri and Y. Oz, Large-N field theories, string theory and gravity, Phys. Rept. 323 (2000) 183 [hep-th/9905111] [INSPIRE].

[8] J. Bros, H. Epstein, M. Gaudin, U. Moschella and V. Pasquier, Anti de Sitter quantum field theory and a new class of hypergeometric identities, Commun. Math. Phys. 309 (2012) 255 [arXiv:1107.5161] [INSPIRE].

[9] V.A. Rubakov, Large and infinite extra dimensions, Phys. Usp. 44 (2001) 871.

[10] P. Brax and C. van de Bruck, Cosmology and brane worlds: A Review, Class. Quant. Grav. 20 (2003) R201 [hep-th/0303095] [INSPIRE].

[11] E. Kiritsis, D-branes in standard model building, gravity and cosmology, Phys. Rept. 421 (2005) 105 [Erratum ibid. 429 (2006) 121-122] [hep-th/0310001] [INSPIRE].

[12] R. Maartens and K. Koyama, Brane-world gravity, Liv. Rev. Relat. 13 (2010) 5.

[13] S.J. Avis, C.J. Isham and D. Storey, Quantum Field Theory in anti-de Sitter Space-Time, Phys. Rev. D 18 (1978) 3565 [inSPIRE].

[14] P. Breitenlohner and D.Z. Freedman, Positive Energy in anti-de Sitter Backgrounds and Gauged Extended Supergravity, Phys. Lett. B 115 (1982) 197 [InSPIRE].

[15] P. Breitenlohner and D.Z. Freedman, Stability in Gauged Extended Supergravity, Annals Phys. 144 (1982) 249 [inSPIRE].

[16] L. Mezincescu and P.K. Townsend, Stability at a Local Maximum in Higher Dimensional Anti-de Sitter Space and Applications to Supergravity, Annals Phys. 160 (1985) 406 [INSPIRE].

[17] A. Ishibashi and R.M. Wald, Dynamics in nonglobally hyperbolic static space-times. 3. Anti-de Sitter space-time, Class. Quant. Grav. 21 (2004) 2981 [hep-th/0402184] [INSPIRE].

[18] A. Ishibashi and R.M. Wald, Dynamics in nonglobally hyperbolic static space-times. 2. General analysis of prescriptions for dynamics, Class. Quant. Grav. 20 (2003) 3815 [gr-qc/0305012] [INSPIRE].

[19] T. Gherghetta and A. Pomarol, Bulk fields and supersymmetry in a slice of AdS, Nucl. Phys. B 586 (2000) 141.

[20] A. Flachi and D.J. Toms, Quantized bulk scalar fields in the Randall-Sundrum brane model, Nucl. Phys. B 610 (2001) 144 [hep-th/0103077] [INSPIRE].

[21] A.A. Saharian, Wightman function and Casimir densities on AdS bulk with application to the Randall-Sundrum brane world, Nucl. Phys. B 712 (2005) 196 [hep-th/0312092] [inSPIRE]. 
[22] C.J. Isham, Twisted Quantum Fields in a Curved Space-Time, Proc. Roy. Soc. Lond. A 362 (1978) 383 [inSPIRE].

[23] C.J. Isham, Spinor Fields in Four-dimensional Space-time, Proc. Roy. Soc. Lond. A 364 (1978) 591 [INSPIRE].

[24] H.B.G. Casimir, On the attraction between two perfectly conducting plates, Proc. K. Ned. Akad. Wet. 51 (1948) 793.

[25] G. Plunien, B. Müller and W. Greiner, The Casimir Effect, Phys. Rept. 134 (1986) 87 [INSPIRE].

[26] E. Elizalde, S.D. Odintsov, A. Romeo, A.A. Bytsenko and S. Zerbini, Zeta Regularization Techniques with Applications, World Scientific, Singapore (1994).

[27] V.M. Mostepanenko, N.N. Trunov, The Casimir Effect and Its Applications, Oxford University Press, Oxford U.K. (1997).

[28] K.A. Milton, The Casimir Effect: Physical Manifestation of Zero-Point Energy, World Scientific, Singapore (2002).

[29] M. Bordag, G.L. Klimchitskaya, U. Mohideen, V.M. Mostepanenko, Advances in the Casimir Effect, Oxford University Press, Oxford U.K. (2009).

[30] D. Dalvit, P. Milonni, D. Roberts and F. da Rosa (eds.), Casimir Physics, Lecture Notes in Physics Vol. 834, Springer-Verlag, Berlin Germany (2011).

[31] E. Elizalde, S.D. Odintsov and A.A. Saharian, Fermionic Casimir densities in anti-de Sitter spacetime, Phys. Rev. D 87 (2013) 084003 [arXiv:1302.2801] [INSPIRE].

[32] A. Flachi, J. Garriga, O. Pujolàs and T. Tanaka, Moduli stabilization in higher dimensional brane models, JHEP 08 (2003) 053 [hep-th/0302017] [INSPIRE].

[33] A. Flachi and O. Pujolàs, Quantum selfconsistency of AdS x Sigma brane models, Phys. Rev. D 68 (2003) 025023 [hep-th/0304040] [INSPIRE].

[34] A.A. Saharian, Wightman function and vacuum fluctuations in higher dimensional brane models, Phys. Rev. D 73 (2006) 044012 [hep-th/0508038] [INSPIRE].

[35] A.A. Saharian, Bulk Casimir densities and vacuum interaction forces in higher dimensional brane models, Phys. Rev. D 73 (2006) 064019 [hep-th/0508185] [INSPIRE].

[36] A.A. Saharian, Surface Casimir densities and induced cosmological constant in higher dimensional braneworlds, Phys. Rev. D 74 (2006) 124009 [hep-th/0608211] [INSPIRE].

[37] E. Elizalde, M. Minamitsuji and W. Naylor, Casimir effect in rugby-ball type flux compactifications, Phys. Rev. D 75 (2007) 064032 [hep-th/0702098] [INSPIRE].

[38] R. Linares, H.A. Morales-Tecotl and O. Pedraza, Casimir force for a scalar field in warped brane worlds, Phys. Rev. D 77 (2008) 066012 [arXiv:0712.3963] [INSPIRE].

[39] M. Frank, N. Saad and I. Turan, The Casimir Force in Randall Sundrum Models with $q+1$ dimensions, Phys. Rev. D 78 (2008) 055014 [arXiv:0807.0443] [INSPIRE].

[40] E.R. Bezerra de Mello, A.A. Saharian and V. Vardanyan, Induced vacuum currents in anti-de Sitter space with toral dimensions, Phys. Lett. B 741 (2014) 155 [arXiv:1410.2860] [INSPIRE].

[41] E.R. Bezerra de Mello and A.A. Saharian, Finite temperature current densities and 
Bose-Einstein condensation in topologically nontrivial spaces,

Phys. Rev. D 87 (2013) 045015 [arXiv: 1211.5174] [INSPIRE].

[42] S. Bellucci, A.A. Saharian and V.M. Bardeghyan, Induced fermionic current in toroidally compactified spacetimes with applications to cylindrical and toroidal nanotubes, Phys. Rev. D 82 (2010) 065011 [arXiv: 1002.1391] [INSPIRE].

[43] S. Bellucci, E.R.B. de Mello and A.A. Saharian, Finite temperature fermionic condensate and currents in topologically nontrivial spaces, Phys. Rev. D 89 (2014) 085002 [arXiv:1312.1686] [INSPIRE].

[44] S. Bellucci, A.A. Saharian and H.A. Nersisyan, Scalar and fermionic vacuum currents in de Sitter spacetime with compact dimensions, Phys. Rev. D 88 (2013) 024028 [arXiv: 1302.1688] [INSPIRE].

[45] S. Bellucci and A.A. Saharian, Fermionic current from topology and boundaries with applications to higher-dimensional models and nanophysics, Phys. Rev. D 87 (2013) 025005 [arXiv: 1207.5046] [INSPIRE].

[46] S. Bellucci, A.A. Saharian and N.A. Saharyan, Casimir effect for scalar current densities in topologically nontrivial spaces, Eur. Phys. J. C 75 (2015) 378 [arXiv:1507.08832] [INSPIRE].

[47] E877 collaboration, J. Barrette et al., Observation of anisotropic event shapes and transverse flow in Au + Au collisions at AGS energy, Phys. Rev. Lett. 73 (1994) 2532 [hep-ex/9405003] [INSPIRE].

[48] S. Bellucci, E.R. Bezerra de Mello, A. de Padua and A.A. Saharian, Fermionic vacuum polarization in compactified cosmic string spacetime, Eur. Phys. J. C 74 (2014) 2688.

[49] A. Mohammadi, E.R. Bezerra de Mello and A.A. Saharian, Induced fermionic currents in de Sitter spacetime in the presence of a compactified cosmic string, Class. Quant. Grav. 32 (2015) 135002 [arXiv:1407.8095] [INSPIRE].

[50] F.C. Khanna, A.P.C. Malbouisson, J.M.C. Malbouisson and A.E. Santana, Quantum field theory on toroidal topology: Algebraic structure and applications, Phys. Rept. 539 (2014) 135 [arXiv: 1409.1245] [INSPIRE].

[51] M.R. Douglas and S. Kachru, Flux compactification, Rev. Mod. Phys. 79 (2007) 733 [hep-th/0610102] [INSPIRE].

[52] U.H. Danielsson, E. Keski-Vakkuri and M. Kruczenski, Vacua, propagators and holographic probes in AdS/CFT, JHEP 01 (1999) 002 [hep-th/9812007] [INSPIRE].

[53] A.P. Prudnikov, Yu.A. Brychkov and O.I. Marichev, Integrals and series, Gordon and Breach, New York U.S.A. (1986).

[54] L. Randall and R. Sundrum, An Alternative to compactification, Phys. Rev. Lett. 83 (1999) 4690 [hep-th/9906064] [InSPIRE].

[55] T. Andrade and D. Marolf, AdS/CFT beyond the unitarity bound, JHEP 01 (2012) 049 [arXiv: 1105.6337] [INSPIRE].

[56] T. Andrade, T. Faulkner and D. Marolf, Banishing AdS Ghosts with a UV Cutoff, JHEP 05 (2012) 011 [arXiv:1112.3085] [INSPIRE].

[57] R. Camporesi, zeta function regularization of one loop effective potentials in anti-de Sitter space-time, Phys. Rev. D 43 (1991) 3958 [INSPIRE]. 
[58] M.M. Caldarelli, Quantum scalar fields on anti-de Sitter space-time, Nucl. Phys. B 549 (1999) 499 [hep-th/9809144] [INSPIRE].

[59] A.A. Saharian, A generalized Abel-Plana formula. Applications to cylindrical functions, Izv. Akad. Nauk Arm. SSR Mat. 22 (1987) 166 [Sov. J. Contemp. Math. Anal. 22 (1987) 70].

[60] A.A. Saharian, The Generalized Abel-Plana Formula with Applications to Bessel Functions and Casimir Effect, Yerevan State University Publishing House, Yerevan (2008).

[61] A.A. Saharian, The Generalized Abel-Plana formula with applications to Bessel functions and Casimir effect, Report No. ICTP/2007/082.

[62] A.A. Saharian, The Generalized Abel-Plana formula with applications to Bessel functions and Casimir effect, arXiv:0708.1187 [INSPIRE].

[63] M. Abramowitz and I. A. Stegun (eds.), Handbook of Mathematical Functions, Dover, New York U.S.A. (1972).

[64] E.R. Bezerra de Mello and A.A. Saharian, Fermionic vacuum polarization by a composite topological defect in higher-dimensional space-time, Phys. Rev. D 78 (2008) 045021 [arXiv:0806.1944] [INSPIRE]. 